\title{
Concentración, diversidad y especialización del cooperativismo en México: aplicación de un Análisis Exploratorio de Datos Espaciales ${ }^{1}$
}

\author{
Carla Carolina Pérez Hernández ${ }^{2}$, Graciela Lara Gómez ${ }^{3}$ y $^{-}$Martín Hernández Calzada ${ }^{4}[$
}

Recibido: 23 de junio de 2021 / Aceptado: 6 de enero de 2022 / Publicado: 9 de febrero de 2022

Resumen. El uso de estadísticas sobre cooperativas es una parte esencial para la formulación de políticas, no obstante,
en México su utilización se ha limitado a estudios descriptivos. Por lo tanto, el propósito inicial de esta investigación es
estimar la concentración, diversidad y especialización del cooperativismo mediante los índices de: Krugman, Hirschman-
Herfindahl y la entropía de Shannon. Mientras que por sector cooperativo se calcula el coeficiente de Gini.
Posteriormente, se ocupa la técnica de redes para hacer una exploración dentro del espacio de los sectores cooperativos.
En un tercer momento se aplica un Análisis Exploratorio de Datos Espaciales (ESDA), con las técnicas denominadas:
Índice de Moran e Índice Local de Asociación Espacial (LISA). La primera explica a nivel general como se encuentra
distribuido el cooperativismo (en materia de concentración, diversidad y especialización), mientras que la segunda explica
localmente el nivel de significancia estadística de los agrupamientos espaciales del mismo fenómeno. Los resultados
muestran que tanto la diversidad como la especialización cooperativa tienen una autocorrelación espacial positiva, es
decir que manifiestan una distribución espacial agrupada, lo anterior es relevante para la formulación de políticas
regionales, ya que se lograron identificar y mapear los lugares que concretamente favorecen y desfavorecen la diversidad
y especialización cooperativa. Finalmente, la técnica de redes muestra que el sector cooperativo Agro expresa una co-
ocurrencia marcada con otros sectores cooperativos, lo cual implica la existencia de una proximidad cognitiva,
organizacional, social o institucional que favorece la posible diversificación cooperativa hacia otros sectores, lo cual
puede ser una vía para ampliar la economía social y solidaria en el país. Palabras clave: Cooperativismo; Especialización; Economía social; Análisis exploratorio de datos espaciales (ESDA); Técnica de redes.

Claves Econlit: A10; C00; L52; O14; O25.

\section{[en] Concentration, diversity and specialization of cooperativism in Mexico: Application of an Exploratory Analysis of Spatial Data}

\begin{abstract}
The use of statistics on cooperatives is an essential part of policy formulation; however, in Mexico its use has been limited to descriptive studies. Therefore, the initial purpose of this research is to estimate the concentration, diversity and specialization of cooperativism using the: Krugman, Hirschman-Herfindahl and Shannon entropy indices. While by cooperative sector the Gini coefficient is calculated. Subsequently, the technique of networks is used to make an exploration within the space of cooperative sectors. In a third moment, an Exploratory Spatial Data Analysis (ESDA) is applied, with the techniques called: Moran's Index and Local Index of Spatial Association (LISA). The first explains at a general level how cooperativism is distributed (in terms of concentration, diversity and specialization), while the second explains locally the level of statistical significance of the spatial groupings of the same phenomenon. The results show that both diversity and cooperative specialization have a positive spatial autocorrelation, therefore, they manifest a grouped spatial distribution, the above is relevant for the formulation of regional policies, since it was possible to identify and map the places that specifically favor and disfavor cooperative diversity and specialization. Finally, the network technique shows that the Agro cooperative sector expresses a co-occurrence with other cooperative sectors, which implies the existence of a cognitive, organizational, social or institutional proximity that favors possible cooperative diversification towards other sectors, which it can be a way to expand the social and solidarity economy in the country. Keywords: Cooperativism; Specialization; Social economy; Exploratory analysis of spatial data (ESDA); Network technique.
\end{abstract}

Sumario. 1. Introducción. 2. Revisión de la literatura. 3. Metodología. 4. Resultados. 5. Conclusiones. 6. Referencias bibliográficas.

1 Proyecto financiado por la Secretaría de Educación Pública (México) con el fondo denominado "Programa para el Desarrollo Profesional Docente" (PROMEP-SEP). Número de acuerdo: 511-6/2020-8587.

2 Universidad Autónoma del Estado de Hidalgo (UAEH), México.

Dirección de correo electrónico: carla_perez@uaeh.edu.mx.

3 Universidad Autónoma de Querétaro (UAQ), México.

Dirección de correo electrónico: glara@uaq.mx.

4 Universidad Autónoma del Estado de Hidalgo (UAEH), México.

Dirección de correo electrónico: martinh@uaeh.edu.mx. 
Cómo citar. Pérez Hernández, C.C.; Lara Gómez, G.; Hernández Calzada, M (2022). Concentración, diversidad y especialización del cooperativismo en México: aplicación de un Análisis Exploratorio de Datos Espaciales. REVESCO. Revista de Estudios Cooperativos, vol. 140, e79941. https://dx.doi.org/10.5209/reve.79941.

\section{Introducción}

La mayoría de los investigadores estudian el alcance de la localización. Las externalidades en las empresas/industrias han empleado índices sintéticos de concentración (por ejemplo, Gini, Herfindahl, Isard, Theil, Ellison-Glaeser, cociente de ubicación, o el índice Hoover-Balassa), todos los cuales miran la distribución espacial de una industria/empresa determinada (medida alternativamente en términos de empleo, producción o número de establecimientos) en un territorio más amplio (Fracasso \& Vittucci Marzetti, 2018).

En este caso, se desarrolló un estudio empírico haciendo uso de los datos del censo del INEGI del año 2019 enfocado en las unidades económicas en los diferentes sectores y cuya denominación jurídica es: "cooperativas". Se calculó por entidad los índices de: Krugman (para estimar la especialización cooperativa), Índice de Hirschman-Herfindahl (para medir la concentración cooperativa), la entropía de Shannon (para calcular la diversidad cooperativa). Posteriormente, se calculan los valores del coeficiente de Gini para cada uno de los 18 sectores cooperativos y aplicando la técnica de redes se logra dibujar el espacio-cooperativo. Finalmente, ocupando los índices inicialmente calculados se corre el Análisis Exploratorio de Datos Espaciales (ESDA) tanto a nivel global como local.

Esta investigación, por ende, tiene dos objetivos principales, el primero es calcular las métricas de concentración, diversidad y especialización del cooperativismo en México; y paralelamente, identificar la red del espacio-cooperativo con el fin de descubrir las coocurrencias entre los diferentes sectores cooperativos, lo que dará luz de las posibilidades de diversificación sectorial cooperativa.

El segundo propósito es realizar un Análisis Exploratorio de Datos Espaciales (ESDA), con las técnicas denominadas: Índice de Moran (I. de Moran) e Índice Local de Asociación Espacial (LISA). El I. de Moran explica a nivel general como se encuentra distribuido el cooperativismo (en materia de concentración, diversidad y especialización), mientras que LISA explica localmente el nivel de significancia estadística de los agrupamientos espaciales del mismo fenómeno.

Lo anterior resulta pertinente en el contexto nacional porque existe una brecha de investigación empírica relacionada con el cooperativismo; ya que se carecen de estudios cuantitativos que muestren la geoestadística de las cooperativas. En parte porque las estadísticas nacionales previamente eran cortas a la hora de censarlas y se sigue adoleciendo de datos abiertos sobre el cooperativismo a nivel sub-nacional y en parte también porque los estudios existentes se limitan a realizar un análisis descriptivo de los datos.

Sosa González et al. (2019), subrayan que, pese a su importancia, las cooperativas en México se han estudiado relativamente muy poco, comparado con las corporaciones privadas y las organizaciones gubernamentales.

Ceballos, Olivares \& Castillo (2016), señalan que las cooperativas a pesar de estar respaldadas por un movimiento internacional, en México se ha dejado de incentivar su formación a nivel nacional y estatal, reflejándose en aspectos como la falta de información y registro de actividades. Reclamando que quienes pretenden investigar ampliamente cualquier fenómeno de este tipo de unidades económicas carecen de información básica como un padrón que las enliste o identifique. Ellos observan un abandono del movimiento cooperativo en nuestro país que se refleja en aspectos como: los cambios de legislación, la existencia de bancos de información cada vez más escuetos, dispersos y difíciles de ubicar, así como el control y número que de ellas se tiene.

No obstante, el INEGI compila la información de las unidades económicas de acuerdo con su marco jurídico, esclareciendo entonces la cantidad de cooperativas a nivel sub-nacional. Segregándolas no sólo por entidad sino también por sector de procedencia. Pese a lo anterior, la consulta de dichas bases de datos sólo es posible mediante el laboratorio de microdatos o procesamiento remoto ${ }^{5}$, el cual no está habilitado para su consulta abierta al público en general.

En ese sentido, es importante recalcar que las estadísticas son una parte esencial de cualquier narrativa política exitosa. Sin datos es poco probable que alguien que intente criticar una política o promover una nueva tenga un impacto y si se busca argumentar el caso para una política que favorezca a las cooperativas, se tiene que demostrar que son importantes para el desarrollo económico y que, en determinadas circunstancias, pueden ser mejores que otros tipos de negocios; y al hacerlo, el apoyo a las cooperativas dará sus frutos (OIT, 2017).

\footnotetext{
Laboratorio de microdatos o Procesamiento remoto para servidores públicos del Estado mexicano, funcionarios de organismos internacionales e investigadores y estudiantes de posgrado, en este se brinda acceso a información con el mayor grado de desagregación para realizar investigaciones académicas y para apoyar la definición, operación o evaluación de política pública. Los resultados se revisan para validar que no violenten los principios de confidencialidad y reserva establecidos en la Ley del Sistema Nacional de Información Estadística y Geográfica. Para tener acceso a este servicio es necesario cumplir con los requisitos que se establecen para cada proyecto estadístico en la sección de microdatos (INEGI, 2021).
} 
A nivel global, es ampliamente reconocido que las estadísticas sobre cooperativas son fundamentales para cuantificar el impacto del cooperativismo en sus miembros y en la economía en su conjunto. Sin embargo, en la mayoría de los países del mundo faltan estadísticas fiables y comparables sobre este rubro (COPAC, 2019).

Este trabajo contribuye por un lado a destapar las estadísticas actuales sobre el cooperativismo (datos abiertos que se pueden consultar en los enlaces de las visualizaciones interactivas incrustadas en este documento) y por otro, a proveer mapeos de las zonas donde se favorece y desfavorece la especialización de este tipo de unidades económicas. Al tiempo que se hace reflexión sobre las rutas de diversificación cooperativa gracias a la técnica de redes.

Derivado de lo anterior, esta investigación fue diseñada para responder las siguientes Preguntas de Investigación (PI):

- $\quad$ (PI-1) ¿Cuáles son los rankings estatales de la concentración, diversidad y especialización sectorial cooperativa? y ¿en qué actividades cooperativas se especializan las entidades mexicanas?

- $\quad$ (PI-2) ¿Cuál de los sectores cooperativos manifiesta el mayor peso de proximidad con otros sectores?

- $\quad$ (PI-3) ¿Cuál de los indicadores espaciales básicos de las cooperativas (especialización, concentración y diversidad) manifiesta una autocorrelación espacial positiva?

Concretamente, se tiene la siguiente hipótesis general:

(H) "La especialización cooperativa tiene una autocorrelación espacial positiva, es decir que la distribución espacial de los valores altos y los valores bajos de dicha variable es agrupada".

Si la hipótesis anterior es cierta entonces sería posible identificar los lugares donde se producen patrones de especialización cooperativa, que pueden ser interpretados como entornos en los que los factores que favorecen la especialización de sociedades cooperativas podrían existir. Asimismo, se podrían identificar otros lugares menos favorables al cooperativismo, que deben ser tenidos en cuenta cuando se trata de estrategias generales y específicas para su revitalización (Pérez y Valiente, 2019).

Claramente los elementos antes mencionados deben tenerse en cuenta a la hora de aplicar estrategias y políticas para el desarrollo regional y promoción y fomento de la economía social y solidaria en el país.

El artículo se desarrolla contemplando los siguientes apartados: a) revisión teórica y contextual del fenómeno de estudio; b) metodología de investigación; c) los resultados y su visualización; d) conclusiones y discusiones.

\section{Revisión de la literatura}

Las cooperativas han sido reconocidas por la Organización de las Naciones Unidas (ONU) como un modelo empresarial que contribuye al desarrollo económico y social de los países. En 2012 fueron distinguidas con la designación del "Año Internacional de las Cooperativas", con el lema "Las empresas cooperativas ayudan a construir un mundo mejor" (ONU, 2012). Además han sido consideradas como una de las organizaciones que inciden fuertemente al cumplimiento de los Objetivos de Desarrollo Sostenible, por su pertinencia en el abatimiento de la pobreza, la creación de empleos, la promoción en la igualdad de género, facilitar el acceso a la salud, por sus aportes a la educación, su contribución a la seguridad alimentaria, a la conservación y saneamiento del agua, el uso de energías alternativas y en general a la gestión sostenible de los recursos naturales (OIT, 2015).

El modelo cooperativo ha demostrado su valía en los contextos locales en los que se desarrollan sus actividades de producción, consumo y servicios, trascendiendo globalmente a través de los beneficios tangibles para las personas con carencias sociales y económicas, pero también como un modelo de negocio que participa eficazmente en el mercado. Algunos datos muestran que el $12 \%$ de la población mundial se encuentra vinculada a los 3 millones de cooperativas que operan en el mundo y que cerca de 1000 millones de personas tienen vinculación con estas, además de que cuentan con unos 100 millones de empleados (ACI, 2018). Un dato igualmente importante es que las 300 cooperativas más importantes, reúnen en su conjunto una renta agregada de 1,6 billones de USD (equivalente al Producto Interno Bruto de España) (OIT, 2015).

En el contexto nacional e internacional la organización más representativa en la Economía Social y Solidaria es "la cooperativa", cuyo objetivo central es la creación de valor social y económico; con esta fórmula se plantea proporcionar respuestas a una necesidad insatisfecha que no está siendo generada en el mercado de manera espontánea (Austin, et al., 2006), por lo que con la creación de valor se persigue favorecer a las personas, principalmente a aquellas que se encuentran en situación de desventaja.

Es por ello que las cooperativas contribuyen a modificar positivamente la vida de los individuos, a través de la obtención de sus aspiraciones. Al respecto Tirole (2017) dice que es claro que el mercado no facilita la convivencia con equidad, pues no propicia la existencia de una estructura de ingresos y beneficios como la sociedad desearía, por lo que es ineludible recuperar la concepción equitativa del bien común, donde los beneficios sean un medio y no un fin, orientando al mercado hacia el beneficio común y logrando la participación eficiente del Estado. 
El cooperativismo representa una doctrina económica y social que nace como una necesidad histórica para asegurar la sobrevivencia y enfrentar problemas comunes. Con tal perspectiva se defiende la idea de que, a través del trabajo, el consumo, la producción y la solidaridad de las personas, es posible resolver problemáticas comunes. Por ende, la cooperativa representa una alternativa viable para contribuir al avance empresarial colectivo y a la cohesión social que permite a sus miembros tomar el mando de su propio desarrollo (Lara Gómez, 2020).

Diversos estudios empíricos se han realizado en el contexto mexicano que exploran el movimiento cooperativo desde distintos ángulos, tales como:

En el estado de Quintana Roo, se realizó un Padrón de Entidades Cooperativas con base en el Directorio Estadístico Nacional de Unidades Económicas 2015 y en datos de la Secretaría de Economía. Detectando que "especialistas y miembros de las organizaciones cooperativas y del movimiento cooperativo en México han afirmado que actualmente este sector se encuentra en una fase de estancamiento, no existen programas en los tres niveles de gobierno que coordinen esfuerzos o políticas a favor de las sociedades cooperativas en particular, salvo el Fideicomiso de apoyo a sociedades cooperativas de ahorro y préstamo. Además de no contar con un registro único de la cantidad de cooperativas existentes que demuestre su importancia, número de miembros o vertiente económica" (Ceballos, Olivares \& Castillo, 2016).

En el estado de Hidalgo, se realizó un artículo que analiza desde la ecología política feminista las estrategias de género implementadas por dos cooperativas dirigidas por mujeres campesinas e indígenas en dicha entidad para acceder y controlar recursos naturales (Soto Alarcón et al., 2020).

En la ciudad de México, Izquierdo (2021) ha documentado los planes y programas que el gobierno de dicha ciudad ha implementado para el fomento de una economía social y solidaria y para la generación de empleos; lo cual ha sido una forma acertada para muchas personas que han quedado fuera del mundo laboral, especialmente para el sector femenino. La autora remarca que actualmente se cuenta con el Programa Apoyo para el Desarrollo de Sociedades Cooperativas de la Ciudad de México y Subprograma de Impulso Cooperativo (2018) así como de diversas acciones que se han ido realizado a través del tiempo como fue la creación de un padrón que para el año 2013 contaba con más de 500 cooperativas, lo que determinaba un modelo de vanguardia en una entidad del país, con la promoción del cooperativismo y de otras formas de economía social y solidaria.

En el mismo contexto, Díaz de León \& Rivera (2019), señalan que el programa “Apoyo para el Desarrollo de Sociedades Cooperativas de la Ciudad de México" (Cooperativas CDMX), es promovido y desarrollado por la Secretaría de Trabajo y Fomento al Empleo de la Ciudad de México (STyFE) durante los años 2015, 2016, 2017 y 2018. Mientras que el objetivo del subprograma Impulso Cooperativo, es como su nombre lo indica, impulsar la formación de nuevas Sociedades Cooperativas, apoyando a grupos sociales de al menos cinco personas que les interese formar una cooperativa, mediante dos apoyos, por un lado, económico para la compra de maquinaria y equipo, así como de servicios que fortalezcan sus procesos productivos y comerciales y; por el otro lado, con capacitación en aspectos cooperativos y organizacionales.

Otro estudio reciente muestra que las cooperativas urbanas, además de lidiar con problemas productivos han visto limitada su expansión debido a las características de su inserción en el mercado. Es por ello por lo que se dieron a la tarea de analizar casos exitosos en la Ciudad de México, especialmente de pequeñas cooperativas urbanas, teniendo como referencia legal que el 23 de mayo de 2012 se creó la Ley de la Economía Social y Solidaria y el Instituto Nacional de la Economía Social (INAES) para cumplir el mandato constitucional de fomentar la ESS a nivel nacional (Luvián, Rosas, Ramírez, 2019).

Por su parte, Hernández Herrera et al (2018), analizan el empoderamiento y cooperativismo femenino, con tres estudios de caso de cooperativas lideradas por mujeres en la Ciudad de México, encontrando evidencia de que las sociedades cooperativas empoderan a las mujeres ya que van más allá de ser solo proyectos empresariales; éstos son capaces de generar beneficios intangibles y de promoción de la autonomía de la mujer. Enfatizando que el cooperativismo es un mecanismo relevante que permite abrigar a las mujeres y las empodera, ya que es en este proceso que conforme pasan los días fortalece su autoestima, su confianza y amor propio esto ayuda a salir adelante ante las vicisitudes de la vida y de sus propios negocios.

En el estado de Baja California, Méndez-Reyes (2017) analiza y describe la formación de cooperativas de transporte de dicha entidad entre 1930 y 1960, vinculando el transporte y la movilidad de pasajeros al desarrollo local y explicando cómo se ajustó la economía, el empleo y el cooperativismo.

En el estado de Oaxaca, Sosa González (2020) describe las diez principales deficiencias que afectan el desarrollo de treinta cooperativas de producción de la Región de la Costa de Oaxaca en México, abarcando las dimensiones normativa-institucional, social, económica, administrativa, técnica y política a partir de considerar que los fenómenos de la realidad son multidimensionales. Asimismo, identifica seis causas estratégicas externas de fondo de los problemas encontrados, siendo estos: 1) Excesiva precariedad económica de los socios cooperativistas; 2) Política educativa alienada, sin programas académicos para la economía social y el emprendimiento; 3) Deficiente arreglo normativo-institucional; 4) Deficientes y limitadas políticas públicas de apoyo a la economía social; 5) Desarrollo de una cultura individualista y oportunista en el suprasistema, y 6) Educación formal escasa y no pertinente a las necesidades de la región. 
En Sinaloa, Alarcón (2018) realiza un análisis documental del dominio, declinación y fracaso de las cooperativas camaroneras en Mazatlán con una serie temporal de 1982 al 2017; y entre las causas del fracaso de las cooperativas de altamar se señalan: el derroche, ineficiencia, corrupción de los directivos, obsolescencia de la flota, falta de subsidios, etc.

En Tabasco, Cano-Salgado et al (2012) relacionaron la innovación social con la capacidad de organización de las cooperativas pesqueras en el municipio de Balancán.

En el estado de Querétaro, Lara Gómez \& Rico Hernández (2011), estudiaron la contribución de las cooperativas de ahorro y crédito al desarrollo local. Demostrando que la organización de cooperativas, la acción voluntaria de sus miembros y en algunos casos el apoyo del Estado, constituyen instrumentos eficaces en la promoción del desarrollo local. Los principales beneficios en favor de los asociados se traducen en el otorgamiento de créditos y de servicios adicionales como becas educacionales y servicios de salud. Pudieron también constatar, que con la práctica de los principios y valores del cooperativismo se facilita el trabajo en equipo y los asociados desarrollan habilidades emprendedoras y directivas que los benefician individual y colectivamente mejorando su calidad de vida.

Es entonces que las cooperativas se manifiestan como el medio a través del cual es posible transformar la realidad de millones de personas, ya que por su conducto se fomenta el emprendimiento de los que menos tienen, con actividades sociales y económicas que contribuyen al desarrollo de las economías locales y que en ocasiones coadyuvan con el Estado en tareas que deben ser asumidas por este (Lara Gómez, 2018).

Rojas-Herrera (2013), realiza un panorama general del cooperativismo agropecuario en México, resaltando que desgraciadamente (en ese entonces) no se sabía a ciencia cierta cuántas cooperativas agropecuarias existían en el país, el último dato oficial proporcionado por la CIFC, indica que hacia 1994 se encontraban registradas 2,753 cooperativas en el giro económico correspondiente a la agricultura, ganadería, caza y silvicultura. Además, indica que en nuestro país, a pesar de que el cooperativismo agropecuario tiene una larga y fecunda tradición histórica, que la legislación invariablemente le ha brindado reconocimiento jurídico, y de que México fue el primer país en realizar una reforma agraria en América Latina en la que las cooperativas estaban llamadas a jugar un papel relevante, hoy en día el cooperativismo agropecuario mexicano exhibe un atraso significativo si se compara con los logros obtenidos por este movimiento social en otras latitudes del mundo.

En el estado de Campeche, Cruz-Coria et al. (2020), exploran las prácticas de la economía solidaria que en la fase de producción y comercialización practican los socios de dos cooperativas artesanales localizadas en dicha entidad.

Cabrera-Ramírez (2015), revisa la trascendencia de las cooperativas en México, mediante un análisis documental del impacto socioeconómico en comunidades rurales. Sus principales resultados son que las cooperativas tienen un papel trascendental para las actividades económicas donde el negocio central es intrínseco en la comunidad y parte de la identidad de ésta; es decir, donde las principales actividades de las cooperativas son parte del conocimiento y dominio popular de la comunidad; asimismo, que los beneficios de una cooperativa fortalecen la actividad económica de la zona y son distribuidos equitativamente. No obstante, a pesar de que la historia registra a las cooperativas aproximadamente desde el siglo XIX, la evolución y presencia de este sistema ha crecido de manera poco significativa, ya que existen pocos registros documentados sobre este tema, los apoyos gubernamentales son insuficientes para impulsar y coadyuvar a la sobrevivencia de este sistema cooperativo en algunas zonas y sectores económicos.

Recientemente, por ejemplo, estudios en España han enmarcado la importancia de analizar la presencia en internet de las cooperativas, identificando una aproximación a la presencia web de las cooperativas en Catalunya, generando una fotografía bastante nítida del estado actual del sector usando el Registro de Cooperativas de Catalunya (Araujo Batlle et al., 2020). Por lo que se considera pertinente medir la presencia cooperativa (concentración, diversidad y especialización) en diferentes contextos tanto geográficos como virtuales.

\subsection{Revisión contextual}

El renacer de la cooperativa es una realidad latente a la que se le debería de prestar mayor atención. El hecho concreto, es que más de mil millones de personas (uno de cada siete habitantes de la Tierra) pertenecen hoy en día a alguna cooperativa. En todo el mundo, centenares de millones de personas compran alimentos a cooperativas, viven en viviendas que han sido construidas en cooperativa y confían sus ahorros a cooperativas financieras. Las cooperativas son el único modelo de negocio que podrá funcionar en una sociedad de costo marginal casi nulo (Rifkin, 2014).

Por otra parte, economistas y geógrafos han siempre compartido el interés en analizar la distribución interregional de las actividades económicas (Cutrini, 2009).

Es bien sabido que las dos dimensiones que caracterizan las economías de aglomeración son la empresarial y la geográfica. Para capturar la interacción entre ellas, los académicos generalmente han recurrido a los conceptos de "concentración" y "especialización". La concentración espacial se relaciona con la ubicación de una empresa/industria en todas las regiones. Especialización, en cambio, se refiere a la distribución de 
empresas/industrias en una región y está inversamente relacionada con el grado de diversificación industrial en la estructura de la economía local. En consecuencia, la concentración aparece como la medida que mejor captura la relación entre las externalidades de localización, por un lado, y el tamaño de las actividades industriales concentradas en un lugar, por otro (Fracasso \& Vittucci Marzetti, 2018).

Contextualmente en México, de acuerdo con el Censo Económico 2014 levantado por el INEGI se contaban con 4,230,745 Unidades Económicas, de las cuales: 9,933 eran Cooperativas. Mientras que en año 2019 las cooperativas censadas ascienden a 11,239 unidades económicas. Entidades como: Jalisco, Ciudad de México, Sinaloa, Sonora y Tabasco, presentan aumentos notorios en la cantidad de cooperativas censadas. Mientras que Oaxaca por ejemplo presenta un decremento notorio (ver tabla 1).

Tabla1. Cooperativas censadas (año 2014 vs. año 2019).

\begin{tabular}{|c|c|c|c|c|}
\hline Descripción & $\begin{array}{c}\text { Cooperativas } \\
2014\end{array}$ & $\begin{array}{c}\text { Cooperativas } \\
2019\end{array}$ & $\begin{array}{l}\text { Variación de } \\
\text { censo a censo }\end{array}$ & $\begin{array}{c}\text { Aumentos } \\
\text { /decrementos }\end{array}$ \\
\hline Aguascalientes & 46 & 75 & 29 & $\uparrow$ \\
\hline Baja California & 117 & 183 & 66 & $\uparrow$ \\
\hline Baja California Sur & 280 & 358 & 78 & $\uparrow$ \\
\hline Campeche & 332 & 367 & 35 & $\uparrow$ \\
\hline Coahuila & 86 & 116 & 30 & $\uparrow$ \\
\hline Colima & 114 & 117 & 3 & $\uparrow$ \\
\hline Chiapas & 765 & 728 & -37 & $\downarrow$ \\
\hline Chihuahua & 115 & 157 & 42 & $\uparrow$ \\
\hline Ciudad de México & 272 & 458 & 186 & $\uparrow$ \\
\hline Durango & 113 & 129 & 16 & $\uparrow$ \\
\hline Guanajuato & 491 & 506 & 15 & $\uparrow$ \\
\hline Guerrero & 630 & 594 & -36 & $\downarrow$ \\
\hline Hidalgo & 203 & 210 & 7 & $\uparrow$ \\
\hline Jalisco & 774 & 990 & 216 & $\uparrow$ \\
\hline México & 261 & 331 & 70 & $\uparrow$ \\
\hline Michoacán & 539 & 519 & -20 & $\downarrow$ \\
\hline Morelos & 103 & 111 & 8 & $\uparrow$ \\
\hline Nayarit & 288 & 256 & -32 & $\downarrow$ \\
\hline Nuevo León & 160 & 238 & 78 & $\uparrow$ \\
\hline Oaxaca & 666 & 560 & -106 & $\downarrow$ \\
\hline Puebla & 322 & 295 & -27 & $\downarrow$ \\
\hline Querétaro & 187 & 261 & 74 & $\uparrow$ \\
\hline Quintana Roo & 98 & 151 & 53 & $\uparrow$ \\
\hline San Luis Potosí & 164 & 180 & 16 & $\uparrow$ \\
\hline Sinaloa & 474 & 674 & 200 & $\uparrow$ \\
\hline Sonora & 408 & 539 & 131 & $\uparrow$ \\
\hline Tabasco & 358 & 555 & 197 & $\uparrow$ \\
\hline Tamaulipas & 245 & 206 & -39 & $\downarrow$ \\
\hline Tlaxcala & 47 & 44 & -3 & $\downarrow$ \\
\hline Veracruz & 719 & 819 & 100 & $\uparrow$ \\
\hline Yucatán & 326 & 351 & 25 & $\uparrow$ \\
\hline Zacatecas & 230 & 161 & -69 & $\downarrow$ \\
\hline Total & 9933 & 11239 & 1,306 & $\uparrow$ \\
\hline
\end{tabular}

Nota: Realizado con datos solicitados al laboratorio de microdatos y procesamiento remoto del INEGI - Censos Económicos 2014 y 2019 por categoría jurídica "cooperativas". Los datos actualmente no están disponibles para su consulta pública en ningún enlace del INEGI. Consulta interactiva en: https://public.dflourish.studio/visualisation/5755963/.

Fuente: Elaboración propia con base en (INEGI, 2014; 2019). 
Por otra parte, entidades como Yucatán, San Luis Potosí, Morelos, Michoacán, Guanajuato, Durango y Colima, prácticamente mantienen el mismo número de unidades económicas cooperativas desde el 2014 hasta la el 2019. Lo cual resulta relevante a la hora de evaluar el fomento a la Economía Social y Solidaria y particularmente lo que respecta a la creación de sociedades cooperativas dentro de dichas regiones, reforzando así la premisa de Ceballos, Olivares \& Castillo (2016), quienes señalan que las cooperativas a pesar de estar respaldadas por un movimiento internacional, en México se ha dejado de incentivar su formación a nivel nacional y estatal. Los movimientos de un censo a otro en cuanto a unidades económicas cooperativas pueden observarse detalladamente en la Figura 1.

Figura. 1. Movimientos de las cooperativas censadas (año 2014 vs. año 2019).

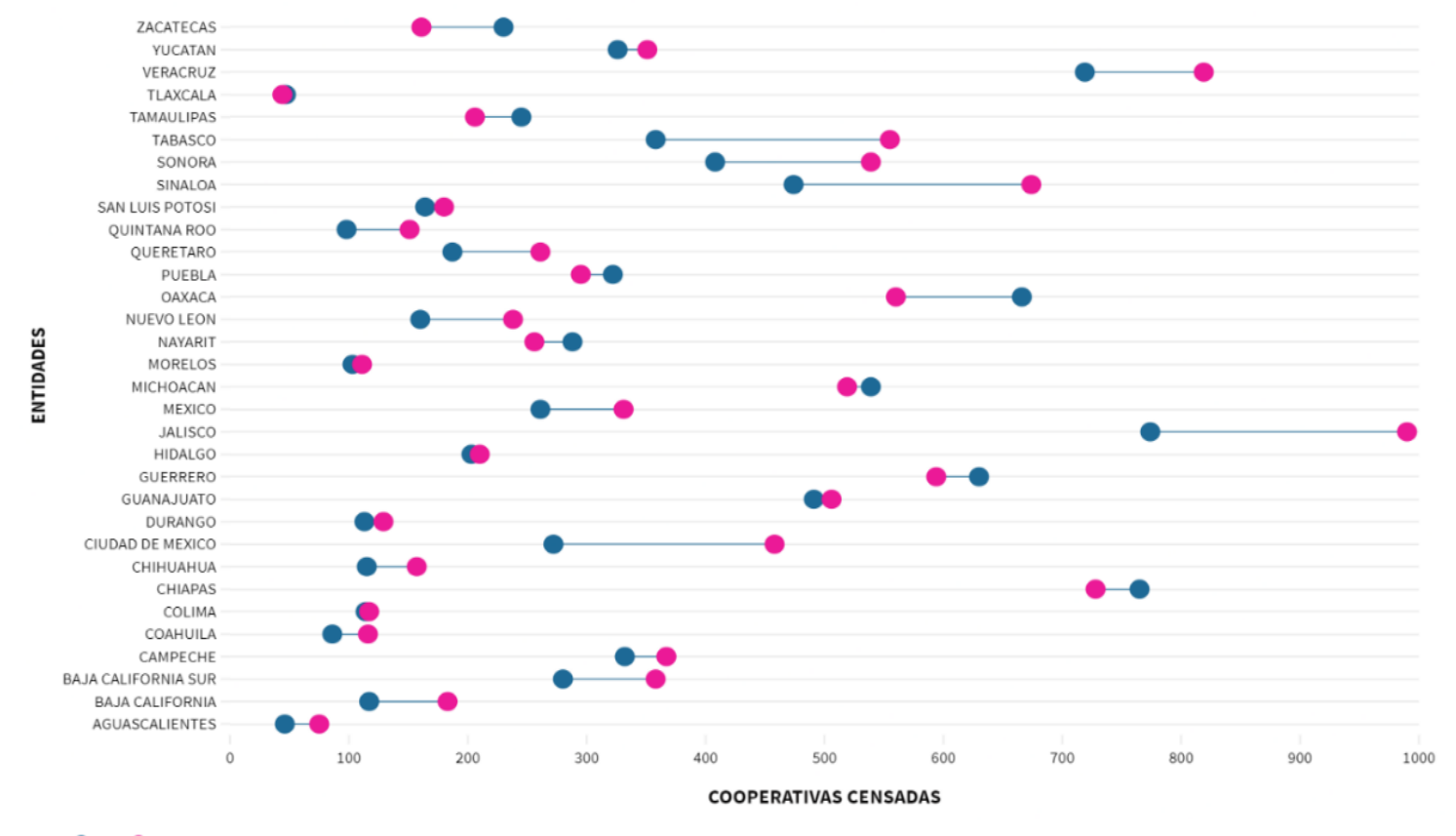

Fuente: Elaboración propia con base en (INEGI, 2014; 2019).

El sector cooperativo con mayor presencia a nivel nacional es el sector primario, es decir aquellas cooperativas dedicadas a la agricultura, cría y explotación de animales, aprovechamiento forestal, pesca y caza. Seguidas de las cooperativas del sector de servicios financieros y en un tercer lugar las cooperativas relacionadas con el transporte, correos y almacenamiento. Del mismo modo, los sectores cooperativos con menor presencia de unidades económicas son las cooperativas de: Energía eléctrica, suministro de agua y de gas, el sector de la Construcción y el sector de información y medios masivos. Tal como se observa en la Tabla 2 . 
Tabla. 2. Total de cooperativas por sector económico en México en 2019.

\begin{tabular}{|l|r|r|}
\hline \multicolumn{1}{|c|}{ NOMBRE } & $\begin{array}{c}\text { COOPERATIVAS } \\
\text { CENSADAS }\end{array}$ & \multicolumn{1}{c|}{ PESO } \\
\hline Agricultura y afines & 4,225 & $37.59 \%$ \\
\hline Minería & 70 & $0.62 \%$ \\
\hline Energía eléctrica, suministro de agua y de gas & 7 & $0.06 \%$ \\
\hline Construcción & 7 & $0.06 \%$ \\
\hline Industrias manufactureras & 540 & $4.80 \%$ \\
\hline Comercio al por mayor & 492 & $4.38 \%$ \\
\hline Comercio al por menor & 768 & $6.83 \%$ \\
\hline Transportes, correos y almacenamiento & 836 & $7.44 \%$ \\
\hline Información en medios masivos & 14 & $0.12 \%$ \\
\hline Servicios financieros y de seguros & 2,468 & $21.96 \%$ \\
\hline Servicios inmobiliarios y de alquiler & 137 & $1.22 \%$ \\
\hline Servicios profesionales, científicos y técnicos & 183 & $1.63 \%$ \\
\hline Manejo de residuos & 181 & $1.61 \%$ \\
\hline Servicios educativos & 91 & $0.81 \%$ \\
\hline Servicios de salud y de asistencia social & 115 & $1.02 \%$ \\
\hline Otros servicios recreativos & 97 & $0.86 \%$ \\
\hline Servicios de alojamiento y alimentos & 413 & $3.67 \%$ \\
\hline Otros servicios & 595 & $5.29 \%$ \\
\hline TOTAL DE COOPERATIVAS CENSADAS & $\mathbf{1 1 , 2 3 9}$ & $\mathbf{1 0 0 \%}$ \\
\hline
\end{tabular}

Fuente: Elaboración propia con base en (INEGI, 2019). Consulta interactiva en: https://public.flourish.studio/visualisation/5748640/.

\section{Metodología}

Se hizo la solicitud de información al laboratorio de microdatos y procesamiento remoto del INEGI con relación al censo económico 2019 referente al cuadro de unidades económicas censadas de acuerdo con su categoría jurídica "cooperativas". A partir de dicha información se procedió a realizar la estimación de los diversos indicadores básicos espaciales, primero se calculó la diversidad cooperativa usando la entropía de Shannon $(\mathrm{H})$; en un segundo momento, la concentración cooperativa fue estimada a través del Índice Hirschman-Herfindahl (HHI), y posteriormente se realizaron los cálculos relacionados con la especialización cooperativa, utilizando para tales efectos el índice Krugman (IK) y la matriz de especialización de Balassa. Finalmente, se estimó la desigualdad de los sectores cooperativos mediante el coeficiente de Gini (CG). Posteriormente, ya con los índices calculados se procedió a aplicar la técnica de redes para graficar el espaciocooperativo con la matriz original de datos (conteo de cooperativas por sector y por entidad). Finalmente, se aplicó un Análisis Exploratorio de Datos Espaciales bajo las técnicas del I. de Moran y LISA para medir la autocorrelación espacial tanto a nivel global como local. Dicho proceso se observa en la Tabla 3. 
Tabla. 3. Proceso metodológico.

\begin{tabular}{|c|c|}
\hline $\begin{array}{c}\text { PASOS } \\
\text { METODOLÓGICOS }\end{array}$ & ESTIMACIONES REALIZADAS \\
\hline $\begin{array}{l}\text { 1. Indicadores } \\
\text { espaciales básicos }\end{array}$ & $\begin{array}{l}\text { Estimaciones: } \\
\text { 1) Herfindahl (Índice de Concentración Cooperativa) } \\
\text { 2) Entropía (Índice de Diversidad Cooperativa) } \\
\text { 3) Krugman (Índice Especialización Cooperativa) } \\
\text { 4) Matriz de especialización de Balassa } \\
\text { 5) Índice de Gini (Desigualdad de los sectores cooperativos) } \\
\text { Justificación de la técnica: La cuestión de la localización industrial o la } \\
\text { especialización geográfica ha pasado rápidamente a primer plano en los últimos } \\
\text { años. Por un lado, la teoría económica ha comenzado a extenderse a un campo } \\
\text { hasta ahora desatendido. Por otro lado, la demanda de investigaciones concretas } \\
\text { se ha visto agudizada por la angustia de las poblaciones "varadas" (no } \\
\text { investigadas) y por la búsqueda de una base para una planificación racional de la } \\
\text { distribución industrial (Hoover, 1936). } \\
\text { Aportación de la técnica: } \\
\text { Este paso nos permite calcular los indicadores clave de la distribución espacial de } \\
\text { las actividades económicas que en este caso son relacionadas con los sectores del } \\
\text { cooperativismo tomando en cuenta las unidades económicas. Aquí buscamos } \\
\text { generar rankings estatales de los indicadores espaciales básicos del } \\
\text { cooperativismo, a saber: concentración, diversidad especialización y desigualdad. }\end{array}$ \\
\hline
\end{tabular}

PREGUNTA DE INVESTIGACIÓN 1: ¿Cuáles son los rankings estatales de la concentración, diversidad especialización cooperativa? ¿En qué actividades cooperativas se especializan las entidades mexicanas?

\section{Estimaciones:}

Teniendo como input la matriz de especialización de Balassa que calcula los cocientes de ubicación de las regiones (de incidencia) - matrices de sectores. El numerador es la participación de un sector cooperativo determinado en una región determinada. El denominador es la participación de este sector en el país, se procede a estimar la red del espacio-cooperativo por medio de la función del

\section{Técnica de Redes} relacionamiento que calcula la relación entre entidades (o bien, sectores, industrias, tecnologías, ...) a partir de su matriz de coincidencia (adyacencia). Se proponen diferentes procedimientos de normalización, en este caso se ocupó la asociación fuerza (Balland, 2017).

Aportación de la técnica: Identificar la red del espacio-cooperativo con el fin de descubrir las coocurrencias (proximidades) entre los diferentes sectores cooperativos, lo que dará luz de las posibilidades de diversificación/especialización sectorial cooperativa.

PREGUNTA DE INVESTIGACIÓN 2: ¿Cuál de los sectores cooperativos manifiesta el mayor peso de proximidad con otros sectores?

\section{Técnicas geoestadísticas de Autocorrelación espacial Global y Local}

1) Índice de Moran (de los indicadores espaciales básicos)

2) LISA (de los indicadores espaciales básicos que resulten no aleatorias)

\section{Justificación de la técnica:}

La autocorrelación espacial es un procedimiento intrínsecamente geográfico que nos puede decir mucho acerca del comportamiento de la información

3. Índices de asociación espacial georreferenciada a diferentes escalas, en particular el tipo de asociación existente entre unidades espaciales vecinas. No obstante, a pesar de su creciente importancia en el marco del análisis exploratorio de datos espaciales, su utilización es reducida en trabajos geográficos que continúan recurriendo a los coeficientes tradicionales y a la estadística descriptiva (Celemín, 2009).

\section{Aportación de la técnica:}

Generar diversos mapeaos relacionados con la especialización y diversificación del cooperativismo en México.

PREGUNTA DE INVESTIGACIÓN 3: ¿Cuál de los indicadores espaciales básicos (especialización, concentración y diversidad) manifiesta una autocorrelación espacial positiva? 
El proceso antes mencionado es desglosado analíticamente en esta sección.

De acuerdo con Balland (2017), es posible ocupar como métrica de diversidad: la entropía, ya que esta otorgará un mayor valor a los sectores cooperativos más pequeños (en términos de unidades económicas), mide tanto la variedad como el equilibrio.

La entropía de Shannon H se puede calcular de la siguiente manera:

$$
H=\sum_{i=1}^{n} P_{i} \log _{2}\left(\frac{1}{p_{i}}\right)
$$

Donde $\mathrm{P}_{\mathrm{i}}$ representa la participación de un determinado sector cooperativo i en la totalidad de cooperativas de la entidad.

Como medida de concentración, se estima el Índice Hirschman-Herfindahl (HHI), ya que este otorga una mayor ponderación a las actividades más grandes y básicamente mide la concentración y el equilibrio de estas (Saviotti \& Frenken, 2008).

$$
H H I=\sum_{i}\left(\frac{E_{i}}{\Sigma_{j}^{N} E_{j}}\right)^{2}
$$

El valor del HHI varía entre 0 y 1 , donde 1 supone una concentración absoluta de las unidades económicas hacia un sector cooperativo i. Por lo tanto, cuanto menor sea el valor, más equilibradas y menos concentradas estarán las actividades.

El índice de especialización cooperativa se calcula haciendo uso del índice de Krugman (Krugman, 1991) a partir de entidades - matrices de sectores cooperativos. Cuanto mayor sea el coeficiente, mayor es la especialización regional cooperativa. Donde $P_{i}^{k}$ es la participación del sector cooperativo $k$ en el total de unidades económicas cooperativas de la región $i$. mientras que $P_{j}^{k}$ es la participación del sector cooperativo k en el total de unidades económicas cooperativas del país $j$.

$$
I K=\sum_{k=1}^{n}\left|P_{i}^{k}-P_{j}^{k}\right|
$$

En otras palabras, el índice será igual a cero si la entidad tiene una estructura de sectores cooperativos similar a la nacional, la cual puede ser considerada como una economía cooperativa poco especializada. Mientras que su valor máximo es dos (en términos absolutos) cuando la entidad no tiene nada en común con la estructura nacional de los sectores cooperativos; por lo tanto, se trataría de una economía cooperativa relativamente especializada.

Este índice es una medida relativa de especialización regional, que, en nuestro caso, compara las unidades económicas de los sectores cooperativos con la estructura nacional cooperativa. Supone que la estructura nacional es un modelo proxy de la economía diversificada para el nivel de desarrollo de la región y el valor muestra la diferencia absoluta entre los sectores cooperativos de las $n$ regiones, o de una región con relación al cooperativismo nacional.

\section{Matriz de especialización cooperativa}

De acuerdo con Hidalgo (2021), solemos organizar datos sobre la geografía de las actividades utilizando matrices que conectan ubicaciones (como países, ciudades o regiones; denotado con el subíndice $c$ y actividades (como productos, industrias o tecnologías; indicado con el subíndice $p$ ). La matriz en este caso utilizada incluye el número de unidades económicas con denominación jurídica "cooperativa" por región y sector cooperativo. En esta notación, tal matriz es: $X_{c p}=$ volumen de actividad (unidades económicas de los sectores cooperativos) en la ubicación c; por consiguiente, la matriz de especialización cooperativa estaría dada por $R_{c p}=X_{c p} X / X_{c} / X_{p}$; donde $R_{c p}$ es la relación entre el valor observado de $\left(X_{c p}\right)$ y el esperado ( $X_{c} / X_{p} / \mathrm{X}$ ) nivel de presencia cooperativa en una ubicación. Las ubicaciones con $R_{c p}>1$ se consideran especializadas en el sector cooperativo $p$.

Finalmente para calcular la desigualdad de la existencia de los sectores cooperativos, hicimos uso del Coeficiente de Gini, ya que de acuerdo con Rosales \& Fuentes (2017) puede calcularse a nivel sectorial y el cual puede estimarse como:

$$
C G=1-\sum_{i=1}^{n}\left(x_{i}-x_{i-1}\right)\left(y_{i}+y_{i-1}\right)
$$

Donde $y_{i}$ representa el porcentaje acumulado de las unidades cooperativas, $x_{i}$ es el lugar que ocupa cada sector cooperativo de acuerdo con las unidades cooperativas censadas. Para el caso de $y_{i}$ se tienen en 
consideración la proporción de las unidades cooperativas que cada sector tiene respecto de las unidades cooperativas censadas a nivel nacional. El índice de Gini mide la desigualdad espacial. Se extiende de 0 (perfecta igualdad) a 1 (perfecta desigualdad).

Al finalizar la estimación de los índices, estos son correlacionados con variables proxy de los resultados de la economía social, a saber: Índice de Desarrollo Humano, Producto Interno Bruto Estatal (PIBE) y el Índice de Educación.

Para concluir, se calcula la matriz de proximidad entre sectores cooperativos mediante una función del análisis de redes que, en este caso, calcula la relación entre entidades y sectores cooperativos a partir de su matriz de co-ocurrencia (adyacencia) y normalizada por fuerza de asociación. "Se proponen diferentes procedimientos de normalización como: fuerza de asociación, coseno, Jaccard y una versión adaptada de la fuerza de asociación a la que nos referimos como índice de probabilidad" (Balland, 2017), tal como se muestra a continuación:

$$
\emptyset_{i i^{\prime}}=\frac{\Sigma_{j} M_{j, i} M_{j . i^{\prime}}}{\max \left(U_{i, 0}, U_{i^{\prime}, 0}\right)}
$$

Donde $M_{j, i}=1$ si la entidad $\mathrm{j}$ tiene un sector cooperativo i con una PCR $\geq 1$ y 0 de otra forma. $U_{i, 0}$ es la ubicuidad del sector cooperativo i.

\section{Análisis Exploratorio de Datos Espaciales}

Dentro del Análisis Exploratorio de Datos Espaciales se realiza inicialmente el cálculo del I de Morán, dicho índice hace referencia a la distribución de una variable en el espacio y da indicio de la (in)existencia de autocorrelación espacial. Se considera este un estadístico global debido a que concentra en un sola cifra el patrón de distribución territorial del fenómeno de estudio (Rogerson y Kedron, 2012). Su representación algebraica queda denotada de la siguiente manera:

$$
I=\frac{n}{\sum_{i=1}^{i=n} w_{j} \sum_{j=1}^{j=n} W_{i j}} * \frac{\sum_{i=1}^{i=n} w_{j} \sum_{j=1}^{j=n} W_{i j}\left(y_{i}-\bar{y}\right)\left(y_{j}-\bar{y}\right)}{\sum_{i=1}^{i=n}\left(y_{i}-\bar{y}\right)^{2}\left(x_{j}-\bar{x}\right)}
$$

En esta fórmula, $n$ significa el número de las unidades (32 entidades) en el mapa, $\mathrm{W}_{\mathrm{ij}}$ es la matriz de distancias que define si las áreas o puntos geográficos, $i$ y $j$, son o no vecinos. Mientras $y$ refleja el valor observado de los índices de concentración del cooperativismo (concentración, diversidad y especialización). Al final el I de Moran toma valores que oscilan entre el -1 y 1; y permite evaluar si los datos se encuentran dispersos, aleatoriamente distribuidos o si se agrupan negativa o positivamente, identificando con ello un patrón de comportamiento de la dependencia espacial que se refleja espacialmente con la conformación de clusters (Espinoza y Rodríguez, 2018).

Además de esta medida global de autocorrelación espacial, existen indicadores locales de Asociación Espacial (LISA) que permiten identificar clusters o agrupaciones espaciales de la variable. Esto significa la posibilidad de obtener entornos favorables (o no favorables) del cooperativismo basado en la agrupación de un número determinado de entidades que pueden compartir sinergias económicas o interterritoriales (Pérez y Valiente, 2019). El I. de Moran Local (I Anselin local de Moran) que se calculó para cada estado de la república mexicana, se utiliza para la obtención de estos conglomerados. Este indicador se define de la siguiente manera (Anselin, 2013):

$$
I_{j}=\frac{\left(y_{i}-\bar{y}\right)}{\sum_{i=1}^{n}\left(y_{i}-\bar{y}\right)^{2 / n}} \sum_{j=1}^{n} W_{i j}\left(y_{j}-\bar{y}\right) \text { para } i \neq j
$$

Esta función intenta calcular el I de Moran local para cada entidad, determinando el grado en el que cada uno es similar a sus vecinos. Esta medida local también está estandarizada de tal manera que si $Z\left(I_{j}\right)$ es estadísticamente significativa, la existencia de un clúster dado o un agrupamiento espa puede confirmarse (Pérez y Valiente, 2019). El mapa resultante muestra las agrupaciones producidas donde un estado presenta un valor alto (o bajo) para la variable que está cerca de aquellos estados que también tienen valores altos (o bajos) (áreas con valores similares) y atípicos o valores extremos (áreas cuyos valores difieren entre sí).

La ventaja del indicador I de Moran local con respecto al I de Moran global es que, además de determinar si existe o no autocorrelación espacial, nos permite identificar dónde se encuentra dicha autocorrelación (Anselin, 2013). 


\section{Resultados}

El Índice de Diversidad Cooperativa (Entropía) se ordena de mayor a menor porque cuanto mayor sea el índice, habrá mayor diversidad de los sectores cooperativos en la entidad. En el top five del ranking se encuentran: México, CDMX, Chihuahua, Quintana Roo y Baja California. En contra parte, las entidades con menor diversidad de sectores cooperativos son: Guerrero, Sonora, Sinaloa, Baja California Sur y Campeche.

El Índice de Concentración Cooperativa (Herfindahl) se ordena de menor a mayor porque cuanto menor sea el valor, más equilibradas y menos concentradas estarán los sectores cooperativos. En el top five del ranking se encuentran: México, CDMX, Chihuahua, Quintana Roo e Hidalgo. Mientras que las entidades con mayor concentración cooperativa son coincidentes con las menos diversas, tal como se observa a continuación.

Figura. 2. Rankings de diversidad y concentración cooperativa en México.

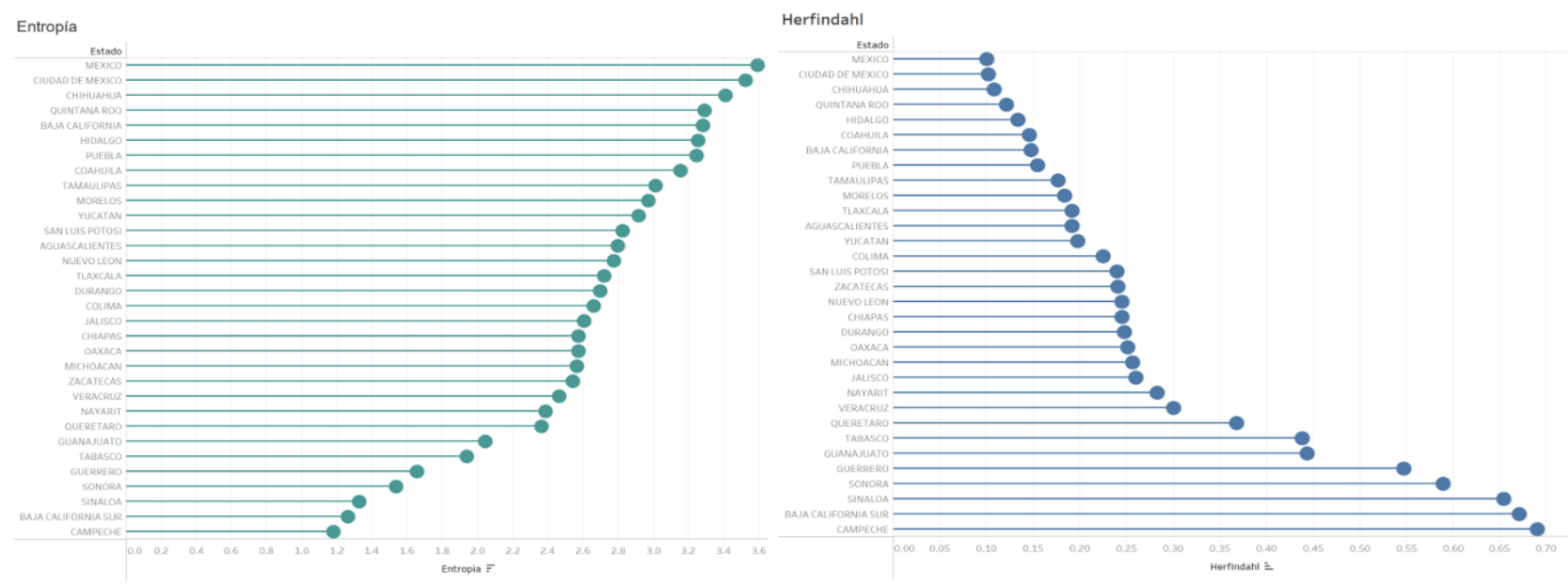

Fuente: Elaboración propia.

Las entidades con mayor cantidad de cooperativas censadas son: Jalisco, Veracruz, Chiapas, Sinaloa y Guerrero. No obstante, ninguna de estas entidades se ubica dentro de las regiones más diversas ni menos concentradas en términos de su actividad cooperativa. En otras palabras, para estados como Chiapas y Guerrero que manifiestan altos índices de marginación, se debería propiciar no sólo el emprendimiento de sociedades cooperativas sino incentivar su diversificación cooperativa, es decir, adherirse a otros sectores cooperativos, los cuales pueden ser adyacentes a los ya existentes.

Medir la diversificación y concentración de los sectores cooperativos, resulta relevante si se considera que la diversificación es vista como aquella que conduce a cambios en las alternativas disponibles en una economía, determinando el número, tipo y calidad de alternativas laborales, bienes de consumo, así como estilo de vida y capital humano. Por lo tanto, es obvio que la diversificación económica y cooperativa están fuertemente relacionadas entre sí y que el estudio de la compleja relación puede proporcionar ideas pertinentes para el desarrollo de políticas públicas, ya que, en teoría, una economía más diversificada puede favorecer el establecimiento de una sociedad pluralista con múltiples ocupaciones y una mejor distribución del poder económico y político (Hartmann, 2014).

Por otra parte, los resultados del Índice de especialización Cooperativa (Krugman) son ordenados de mayor a menor porque cuanto mayor sea el coeficiente, mayor es la especialización regional cooperativa en la entidad. Los primeros 3 lugares del ranking se encuentran: CDMX, Nuevo León y Querétaro. El caso de la Cuidad de México, por ejemplo, presenta una especialización en los sectores cooperativos de: industrias manufactureras, comercio al por mayor, comercio al por menor, información en medios masivos, servicios inmobiliarios y de alquiler, servicios profesionales, científicos y técnicos, manejo de residuos, servicios educativos, servicios de salud y de asistencia social, otros servicios recreativos, servicios de alojamiento y alimentos y otros servicios. Tal como se observa en la Figura 3. 
Figura. 3. Ranking de especialización y matriz de especialización cooperativa en México.
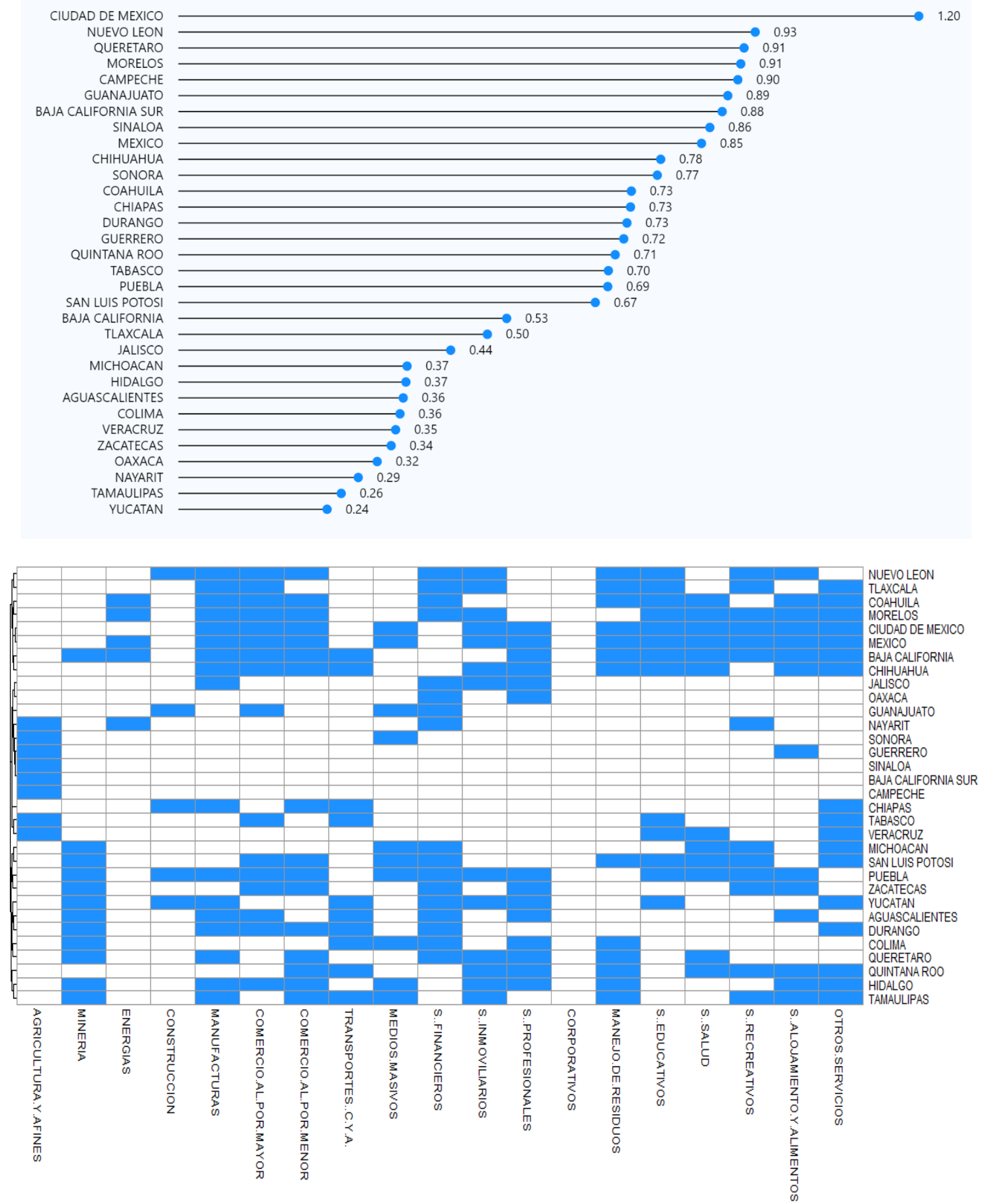

Fuente: Elaboración propia.

Llama la atención el caso de los estados cuya especialización cooperativa es el sector 11 "Agro" (Veracruz, Sonora, Guerrero, Nayarit, Zacatecas, Baja California Sur, Campeche y Sinaloa) a pesar de no tener una alta diversidad de sectores cooperativos con una ventaja comparativa revelada (recuadros azules), es claro que acaparan el sector Agro-cooperativista y en el caso de Campeche y Baja California Sur, alcanzan a ocupar buenas posiciones en el ranking de especialización. Es importante recordar, que la suma de sectores de 
especialización cooperativa por entidad (recuadros azules), enmarcan la diversidad, la cual nosotros estimamos mediante el índice de Shannon, y en este caso, el Índice de Krugman hace referencia a la especialización regional cooperativa.

En ese sentido, es importante resaltar que el objetivo de la especialización inteligente no es hacer que la estructura económica de las regiones sea más especializada (es decir, menos diversificada), sino aprovechar las fortalezas existentes, identificar oportunidades ocultas y generar plataformas novedosas sobre las cuales las regiones pueden construir una ventaja competitiva de alto valor (Balland et al., 2019).

En la Tabla 4 se muestran los índices de diversidad, concentración y especialización cooperativa. Si se pretende buscar una especialización inteligente cooperativa, esta dependerá de reconocer inicialmente en verificar cuál de estas métricas tiene una repercusión estadísticamente significativa sobre los resultados esperados de la economía social. Y posteriormente, identificar región por región cuales son las fortalezas y oportunidades para ir incrementando ya sea, su nivel de diversidad cooperativa, la concentración o desconcentración cooperativa o bien, la especialización cooperativa mediante la diversificación inteligente. Como se verá más adelante, es la especialización cooperativa la que refleja de mejor manera los beneficios esperados de la economía social, lo cual concuerda con Tacchela et al. (2012), quien sostiene que hay evidencia del papel de liderazgo de la diversificación con respecto a la ventaja competitiva (especialización).

Tabla 4. Índices del Cooperativismo en México (año 2019)

\begin{tabular}{|c|c|c|c|c|}
\hline ID & ENTIDAD & $\begin{array}{c}\text { HERFINDAHL } \\
\text { (ÍNDICE DE } \\
\text { CONCENTRACIÓN } \\
\text { COOPERATIVA) }\end{array}$ & $\begin{array}{c}\text { ENTROPÍA } \\
\text { (ÍNDICE DE } \\
\text { DIVERSIDAD } \\
\text { COOPERATIVA) }\end{array}$ & $\begin{array}{l}\text { KRUGMAN (ÍNDICE } \\
\text { ESPECIALIZACIÓN } \\
\text { COOPERATIVA) }\end{array}$ \\
\hline 1 & Aguascalientes & 0.19 & 2.80 & 0.36 \\
\hline 2 & Baja California & 0.15 & 3.28 & 0.53 \\
\hline 3 & Baja California Sur & 0.67 & 1.26 & 0.88 \\
\hline 4 & Campeche & 0.69 & 1.18 & 0.90 \\
\hline 5 & Chiapas & 0.25 & 2.58 & 0.73 \\
\hline 6 & Chihuahua & 0.11 & 3.41 & 0.78 \\
\hline 7 & Ciudad de México & 0.10 & 3.52 & 1.20 \\
\hline 8 & Coahuila & 0.15 & 3.16 & 0.73 \\
\hline 9 & Colima & 0.23 & 2.66 & 0.36 \\
\hline 10 & Durango & 0.25 & 2.70 & 0.73 \\
\hline 11 & Guanajuato & 0.44 & 2.04 & 0.89 \\
\hline 12 & Guerrero & 0.55 & 1.66 & 0.72 \\
\hline 13 & Hidalgo & 0.13 & 3.26 & 0.37 \\
\hline 14 & Jalisco & 0.26 & 2.61 & 0.44 \\
\hline 15 & México & 0.10 & 3.59 & 0.85 \\
\hline 16 & Michoacán & 0.26 & 2.57 & 0.37 \\
\hline 17 & Morelos & 0.18 & 2.97 & 0.91 \\
\hline 18 & Nayarit & 0.28 & 2.39 & 0.29 \\
\hline 19 & Nuevo León & 0.25 & 2.78 & 0.93 \\
\hline 20 & Oaxaca & 0.25 & 2.57 & 0.32 \\
\hline 21 & Puebla & 0.15 & 3.25 & 0.69 \\
\hline 22 & Querétaro & 0.37 & 2.36 & 0.91 \\
\hline 23 & Quintana Roo & 0.12 & 3.29 & 0.71 \\
\hline 24 & San Luis Potosí & 0.24 & 2.83 & 0.67 \\
\hline 25 & Sinaloa & 0.65 & 1.33 & 0.86 \\
\hline 26 & Sonora & 0.59 & 1.54 & 0.77 \\
\hline 27 & Tabasco & 0.44 & 1.94 & 0.70 \\
\hline 28 & Tamaulipas & 0.18 & 3.01 & 0.26 \\
\hline 29 & Tlaxcala & 0.19 & 2.72 & 0.50 \\
\hline 30 & Veracruz & 0.30 & 2.47 & 0.35 \\
\hline 31 & Yucatán & 0.20 & 2.92 & 0.24 \\
\hline 32 & Zacatecas & 0.24 & 2.54 & 0.34 \\
\hline
\end{tabular}

Nota: Valores de referencia: El Índice de Diversidad Cooperativa (Entropía) revela que cuanto mayor sea éste, habrá mayor diversidad de los sectores cooperativos en la entidad. El valor del Herfindahl varía entre 0 y 1, donde 1 supone una concentración absoluta de las unidades económicas hacia un sector cooperativo i. Mientras que, en el valor de Krugman, el índice será igual a cero si la entidad tiene una estructura de sectores cooperativos similar a la nacional, la cual puede ser considerada como una economía cooperativa poco especializada. 
Finalmente, con lo que respecta al análisis de redes, la Figura 4 muestra el espacio de los sectores cooperativos en México para el año 2019, con diferentes atributos, el primero de ellos muestra en el tamaño de los nodos la cantidad de cooperativas por sector; donde es evidente que el sector que acapara la cuantía de cooperativas es el sector agro, servicios financieros y comercio al por menor. En una segunda visualización el tamaño de los nodos esta dado por el valor del PIB por sector, en donde son las industrias manufactureras (cooperativas y no cooperativas) las que abonan en mayor medida al PIB, seguido del sector de servicios inmobiliarios y comercio al por menor. Con lo que respecta la Coeficiente Gini, se observa que los sectores más desigualmente distribuidos son: Energía eléctrica, suministro de Agua y de gas; construcción, información en medios masivos y curiosamente el Sector Agro, esto último implica que dicho sector a pesar de ser el de mayor cuantía cooperativa está presente en pocas entidades.

Figura. 4. Espacio de los Sectores Cooperativos en México (año 2019).

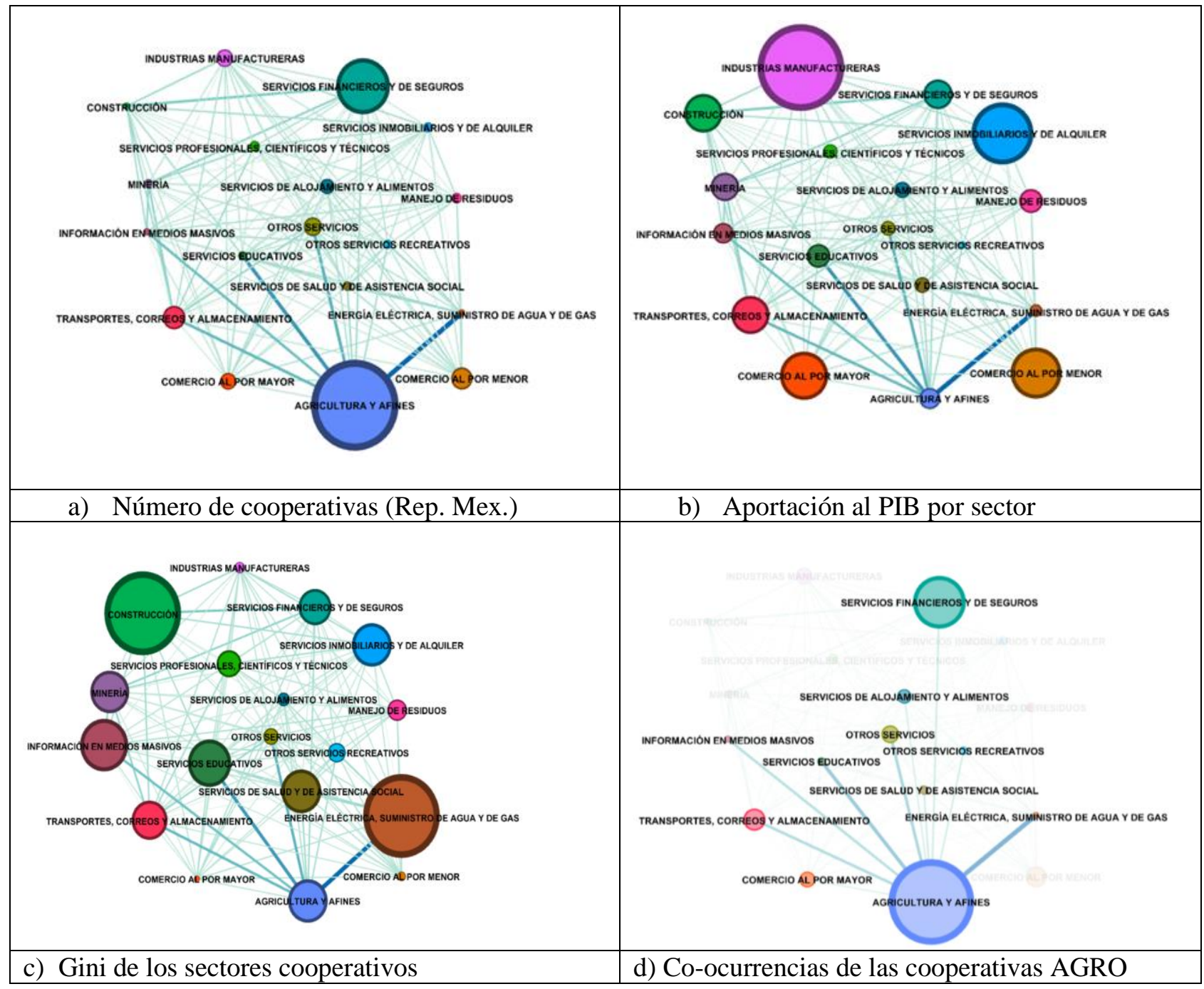

Fuente: Elaboración propia.

Los altos valores del coeficiente de Gini, en la Figura 4 inciso c, expresan un alto grado de concentración de las actividades antes mencionadas, el sector cooperativo más desigual (el más concentrado) en su distribución de unidades económicas es también uno de los que se proyecta pueda tener mayores beneficios socio-ambientales y por lo tanto se debería buscar desconcentrar dicho sector, ya que "las cooperativas de energía sustentable pueden detonar co-beneficios asociados al desarrollo de proyectos de energía renovable, principalmente para los sociales y la comunidad. Entre estos se incluye la reducción de la pobreza energética, acceso a energía eléctrica sustentable y de calidad y la democratización de la energía" (INAES, 2020).

Llama la atención que al dibujar el grosor de las aristas conforme al peso de proximidad que deriva de la matriz de adyacencias, el Sector cooperativo Agro, muestra vínculos fuertes con: el sector energético, servicios educativos, otros servicios, transportes, correos y almacenamiento (principalmente) ; dicho hallazgo resulta relevante si se considera que dicha ligazón se entabla con sectores cooperativos con los que se tiene una co- 
ocurrencia fuerte, es decir que dada la actual estructura cooperativa, estos sectores tienen a estar relacionados con lo que respecta a su presencia en unidades económicas.

La Figura 4, es reveladora si se considera que dicha red muestra el relacionamiento de la presencia de los diferentes sectores cooperativos a nivel nacional, en otras palabras, por ejemplo, se comprueba empíricamente que el sector cooperativo agro, tiene un proximidad apreciable con el sector cooperativo energético y en ese sentido es necesario reflexionar que según Hidalgo et al. (2018) lo que a veces es contraintuitivo es que el principio de relación no se trata de una especialización excesiva. Se trata de comprender los caminos únicos que conducen a la diversificación. Al igual que nuestra comprensión de la gravedad está vinculada a nuestro sueño de volar, nuestra comprensión del relacionamiento está vinculado a nuestro deseo de romper las ligaduras de la dependencia del camino. Entonces, el principio del relacionamiento también sugiere que la política industrial no debe centrarse únicamente en identificar industrias prometedoras, sino en identificar mecanismos que faciliten los flujos de conocimiento entre los sectores y la región.

En este sentido, se subraya la importancia de la proximidad no sólo geográfica sino también la proximidad cognitiva, organizacional, social o institucional que favorece la existencia del cooperativismo en diversos sectores. El espacio de los sectores cooperativos es un dibujo de la co-aglomeración de las unidades económicas cooperativas en los diversos sectores económicos existentes (sector primario, secundario y terciario).

Con lo que respecta a la aplicación del Análisis Exploratorio de Datos Espaciales, se reportan los siguientes resultados. La herramienta Autocorrelación espacial (I de Moran global) mide la autocorrelación espacial basada en las ubicaciones y los valores de las entidades simultáneamente. Dado un conjunto de entidades (estados de la república mexicana) y un atributo asociado (en este caso la concentración, diversidad y especialización cooperativa); se evaluó en cada caso si el patrón expresado está agrupado, disperso o es aleatorio. La herramienta Autocorrelación espacial (I de Moran) es una estadística deductiva, lo que significa que los resultados del análisis siempre se interpretan dentro del contexto de la hipótesis nula. Para la estadística I de Moran global, la hipótesis nula establece que el atributo que se analiza está distribuido en forma aleatoria entre las entidades del área de estudio; es decir, los procesos espaciales que promueven el patrón de valores observado constituyen una opción aleatoria (ArcGIS, 2021).

Para el primer atributo Herfindahl (Índice de Concentración Cooperativa) se tiene un puntaje z de 1.28, por lo que se puede deducir que el patrón de distribución espacial de la concentración cooperativa no parece ser significativamente diferente al aleatorio.

Para el atributo Entropía (Índice de Diversidad Cooperativa) se tiene un puntaje z de 1.66 por lo que hay menos del $10 \%$ de probabilidad de que este patrón agrupado pueda ser el resultado de una probabilidad aleatoria. Por lo tanto, dado que el valor $\mathrm{P}$ es estadísticamente significativo y la puntuación z es positiva. Se puede rechazar la hipótesis nula y deducir que en lo general la distribución espacial de los valores altos y los valores bajos de la diversidad cooperativa está más agrupada de lo que se esperaría si los procesos espaciales subyacentes fueran aleatorios.

Para el atributo Krugman (Índice Especialización Cooperativa) se tiene un puntaje z de 1.67, entonces hay menos del $10 \%$ de probabilidad de que este patrón agrupado pueda ser el resultado de una probabilidad aleatoria. Por lo tanto, dado que el valor $\mathrm{P}$ es estadísticamente significativo y la puntuación z es positiva. Se puede rechazar la hipótesis nula y deducir que en lo general la distribución espacial de los valores altos y los valores bajos de la especialización cooperativa está más agrupada de lo que se esperaría si los procesos espaciales subyacentes fueran aleatorios. Para más detalles ver Tabla 5. 
Tabla. 5. Resultados I. de Moran (Autocorrelación espacial Global)

\begin{tabular}{|c|c|c|c|}
\hline ÍNDICES & RESULTADOS & INTERPRETACIÓN & PATRÓN GENERAL \\
\hline $\begin{array}{l}\text { Herfindahl } \\
\text { (Índice de } \\
\text { Concentración } \\
\text { Cooperativa) }\end{array}$ & $\begin{array}{c}\text { Moran's Index: } \\
0.078381 \\
\text { Expected Index: } \\
-0.032258 \\
\text { Variance: } \\
0.007365 \\
\text { z-score: } \\
1.289222 \\
\text { p-value: } \\
0.197321\end{array}$ & $\begin{array}{c}\text { Dado el puntaje } \mathrm{z} \text { de } \\
\text { 1.28922235412, el patrón no } \\
\text { parece ser significativamente } \\
\text { diferente al aleatorio. }\end{array}$ & Random \\
\hline $\begin{array}{l}\text { Entropía (Índice } \\
\text { de Diversidad } \\
\text { Cooperativa) }\end{array}$ & $\begin{array}{c}\text { Moran's Index: } \\
0.111620 \\
\text { Expected Index: } \\
-0.032258 \\
\text { Variance: } \\
0.007455 \\
\text { z-score: } \\
1.666337 \\
\text { p-value: } \\
0.095646^{* * *}\end{array}$ & $\begin{array}{c}\text { Dado el puntaje } \mathrm{z} \text { de } \\
1.66633721039 \text {, hay menos del } \\
10 \% \text { de probabilidad de que } \\
\text { este patrón agrupado pueda ser } \\
\text { el resultado de una } \\
\text { probabilidad aleatoria. }\end{array}$ & red \\
\hline $\begin{array}{c}\text { Krugman } \\
\text { (Índice } \\
\text { Especialización } \\
\text { Cooperativa) }\end{array}$ & $\begin{array}{c}\text { Moran's Index: } \\
0.113997 \\
\text { Expected Index: } \\
-0.032258 \\
\text { Variance: } \\
0.007582 \\
\text { z-score: } \\
1.679668 \\
\text { p-value: } \\
0.093022 * * *\end{array}$ & $\begin{array}{c}\text { Dado el puntaje } \mathrm{z} \text { de } \\
1.67966834114 \text {, hay menos del } \\
10 \% \text { de probabilidad de que } \\
\text { este patrón agrupado pueda ser } \\
\text { el resultado de una } \\
\text { probabilidad aleatoria. }\end{array}$ & Clustered \\
\hline
\end{tabular}

Nota: Significancia: $0.01 * 0.05 * * .10 * * *$. Los valores de referencia: Los valores oscilan entre -1 (indicando dispersión perfecta) a 1 (correlación perfecta). Un valor de cero indica un patrón espacial aleatoria. Para las pruebas de hipótesis estadísticas, los valores de Moran I pueden ser transformados a la Z-score en el que los valores superiores a 1.96 o menor que -1.96 indican autocorrelación espacial que es significativo al nivel del $5 \%$.

Fuente: Elaboración propia.

Los resultados obtenidos para la I de Moran aplicada a la diversidad y especialización de cooperativas muestran la existencia de autocorrelación espacial global en ambos casos; es decir, la diversidad y especialización de los sectores cooperativos (en términos relativos) en una entidad dada está influenciada generalmente por el grado de diversidad y especialización en los estados contiguos. Además, en ambos casos la autocorrelación global es positiva, lo que indica que los valores altos (o bajos) del índice de diversidad y especialización cooperativa en un estado dado sugieren valores altos (o bajos) de esta misma variable en estados vecinos o contiguos. Por tal motivo se procede a mapear (Figura 5) dichas variables para identificar los clusters altos y bajos por quintiles. 
Figura. 5. Mapas de la diversidad y especialización cooperativa por quintiles.
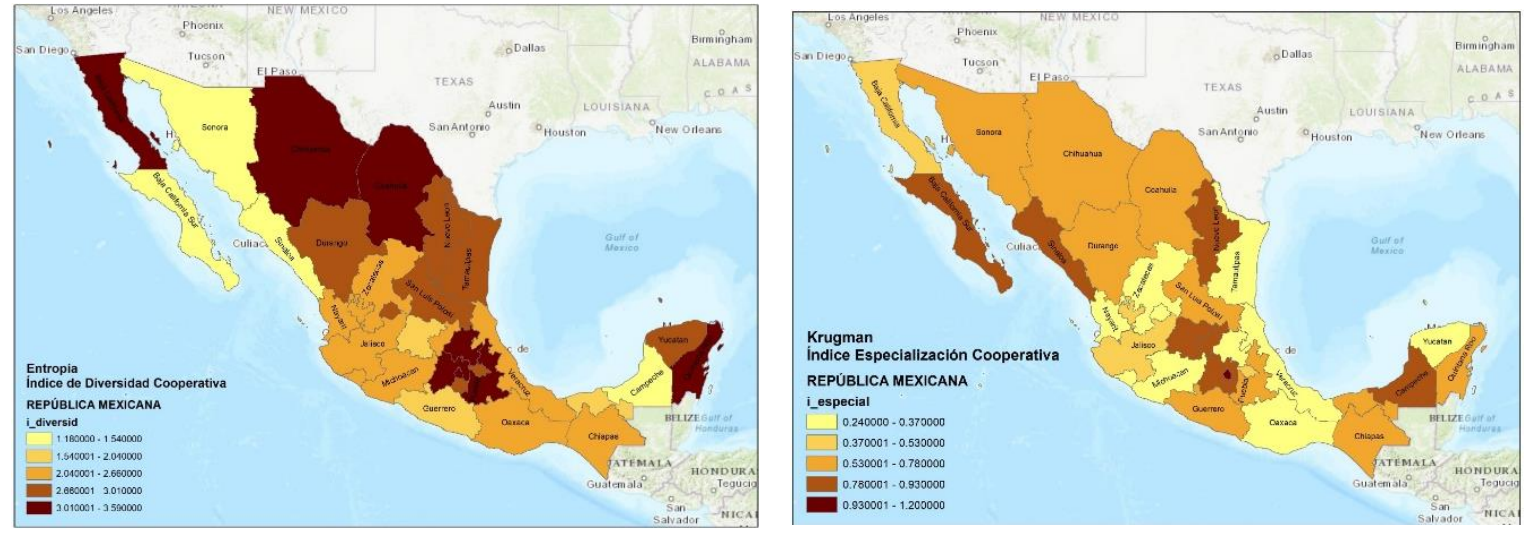

Fuente: Elaboración propia.

Sin embargo, lo que se acaba de describir ocurre a nivel general, es decir, en toda la república mexicana. Por este motivo, y dada la diversidad territorial existente en el país, LISA se ha aplicado con el fin de detectar agrupaciones estadísticamente significativas. Los resultados más notables se discuten continuación.

Para la variable de Entropía (Indice de Diversidad Cooperativa), la autocorrelación espacial no existe en 27 del total de 32 estados, lo que implica que no responde de forma significativa local a un patrón de comportamiento. Hay 2 estados con una alta diversidad cooperativa rodeadas de otras que también muestran altas proporciones (HH: alto-alto, también denominados hot spot); es decir, se trata de entornos que favorecen la diversidad cooperativa situados en el centro del país, particularmente: Estado de México y la Ciudad de México.

Respecto a los clusters espaciales con bajos valores de diversidad del cooperativismo (LL: bajo-bajo, también denominados cold spot), esto se localizó significativamente en: Baja California Sur, lugar donde los sectores predominantes son agricultura y afines y transportes. En cuanto a los valores atípicos o autocorrelación espacial negativa (bajo-alto y alto-bajo), es decir, en este caso los estados con valores bajos para esta variable y rodeados de otros con valores altos o viceversa, hay un total de 2 (Chihuahua y Guerrero). Esto puede significar que, a pesar de la existencia de algunos estados con un notable nivel de diversidad cooperativa (valores atípicos), esta diversificación no se ha extendido con éxito a zonas contiguas, posiblemente debido a la falta de cooperación o de estructura de apoyo por parte de los distintos sistemas de producción locales. Sin embargo, de acuerdo con Pérez y Valiente (2019), esto significa una oportunidad o potencial latente para la generación de sinergias o una fuerza dinámica de atracción hacia cooperativismo y su diversificación sectorial. El estado de Chihuahua resultó como un alto atípico (HL) mientras que Guerrero se muestra como un atípico bajo (LH). Lo antes mencionado se presenta en la Tabla 6.

Tabla.6. Resultados de LISA para la variable de Entropía (Índice de Diversidad Cooperativa).

\begin{tabular}{|r|r|r|r|l|l|l|}
\hline ID & $\begin{array}{c}\text { MORAN } \\
\text { LOCAL }\end{array}$ & Z SCORE & P-VALUE & TIPO & \multicolumn{1}{|c|}{ ENTIDAD } & INTERPRETACIÓN \\
\hline $\mathbf{1}$ & 0.000008 & 3.393618 & 0.00069 & LL & Baja California Sur & Cold spot \\
\hline $\mathbf{7}$ & -0.00001 & -3.162227 & 0.001566 & HL & Chihuahua & Atípico alto \\
\hline $\mathbf{1 1}$ & -0.000032 & -2.757837 & 0.005819 & LH & Guerrero & Atípico bajo \\
\hline $\mathbf{2 3}$ & 0.000058 & 2.303982 & 0.021224 & HH & Ciudad de México & Hot spot \\
\hline $\mathbf{2 4}$ & 0.000049 & 2.284079 & 0.022367 & HH & Estado de México & Hot spot \\
\hline
\end{tabular}

Nota: Las demás entidades no resultaron estadísticamente significativas.

Fuente: Elaboración propia.

Para la variable de Krugman (Índice Especialización Cooperativa) hay 2 entidades con una alta especialización cooperativa rodeadas de otras que también muestran altas proporciones (HH: alto-alto, también denominados hot spot); es decir, se trata de entornos que favorecen la especialización cooperativa situados en el centro del país, particularmente: Morelos y la Ciudad de México regiones que al observar su matriz de especialización tienen una alta especialización en la mayoría de los sectores. Tal como se observa en la Tabla 7. 
Tabla. 7. Resultados de LISA para la variable de Krugman (Índice Especialización Cooperativa).

\begin{tabular}{|r|r|r|r|l|l|l|}
\hline ID & $\begin{array}{c}\text { MORAN } \\
\text { LOCAL }\end{array}$ & Z SCORE & P-VALUE & TIPO & ENTIDAD & INTERPRETACIÓN \\
\hline $\mathbf{1 6}$ & 0.000041 & 1.962371 & 0.049719 & HH & Morelos & Hot spot \\
\hline $\mathbf{1 7}$ & 0.00002 & 2.532584 & 0.011323 & LL & Nayarit & Cold spot \\
\hline $\mathbf{2 3}$ & 0.000053 & 2.121395 & 0.033889 & HH & Ciudad de México & Hot spot \\
\hline
\end{tabular}

Nota: Las demás entidades no resultaron estadísticamente significativas.

Fuente: Elaboración propia.

Respecto a los clusters espaciales con bajos valores de especialización (LL: bajo-bajo, también denominados cold spot), esto se localizó significativamente en: Nayarit, lugar donde los sectores predominantes son únicamente la agricultura y otros servicios recreativos (ver tabla 8 y Figura 6).

Tabla. 8. Fragmento de la Matriz de Especialización Cooperativa.

\begin{tabular}{|c|c|c|c|c|}
\hline \multicolumn{2}{|c|}{ SECTORES COOPERATIVOS } & \multirow{2}{*}{$\begin{array}{c}\text { CIUDAD } \\
\text { DE } \\
\text { MEXICO } \\
0 \\
\end{array}$} & \multirow{2}{*}{$\begin{array}{c}\text { MORELOS } \\
0 \\
\end{array}$} & \multirow{2}{*}{$\begin{array}{c}\text { NAYARIT } \\
1 \\
\end{array}$} \\
\hline Sector 11 & Agricultura y afines & & & \\
\hline Sector 21 & Minería & 0 & 0 & 0 \\
\hline Sector 22 & $\begin{array}{l}\text { Energía eléctrica, suministro de agua y de } \\
\text { gas }\end{array}$ & 0 & 1 & 1 \\
\hline Sector 23 & Construcción & 0 & 0 & 0 \\
\hline Sector 31-33 & Industrias manufactureras & 1 & 1 & 0 \\
\hline Sector 43 & Comercio al por mayor & 1 & 1 & 0 \\
\hline Sector 46 & Comercio al por menor & 1 & 1 & 0 \\
\hline Sector $48-49$ & Transportes, correos y almacenamiento & 0 & 0 & 0 \\
\hline Sector 51 & Información en medios masivos & 1 & 0 & 0 \\
\hline Sector 52 & Servicios financieros y de seguros & 0 & 1 & 1 \\
\hline Sector 53 & Servicios inmobiliarios y de alquiler & 1 & 1 & 0 \\
\hline Sector 54 & $\begin{array}{l}\text { Servicios profesionales, científicos y } \\
\text { técnicos }\end{array}$ & 1 & 0 & 0 \\
\hline Sector 56 & Manejo de residuos & 1 & 0 & 0 \\
\hline Sector 61 & Servicios educativos & 1 & 1 & 0 \\
\hline Sector 62 & Servicios de salud y de asistencia social & 1 & 1 & 0 \\
\hline Sector 71 & Otros servicios recreativos & 1 & 1 & 1 \\
\hline Sector 72 & Servicios de alojamiento y alimentos & 1 & 1 & 0 \\
\hline Sector 81 & Otros servicios & 1 & 1 & 0 \\
\hline & $\begin{array}{l}\text { SECTORES DE } \\
\text { ESPECIALIZACIÓN }\end{array}$ & 12 & 11 & 4 \\
\hline & CLUSTER SIGNIFICATIVO & $\begin{array}{c}\text { HH } \\
\text { (hot spot) }\end{array}$ & $\begin{array}{c}\text { HH } \\
\text { (hot spot) }\end{array}$ & $\begin{array}{c}\text { LL } \\
\text { (cold spot) }\end{array}$ \\
\hline
\end{tabular}

Nota: Los 1 representan especialización en dicho sector mientras que los 0 denotan falta de especialización en el sector.

Fuente: Elaboración propia. 
Figura.6. Mapas de LISA (I. de Moran Local).

LISA para la variable de Entropía
(Índice de Diversidad Cooperativa)

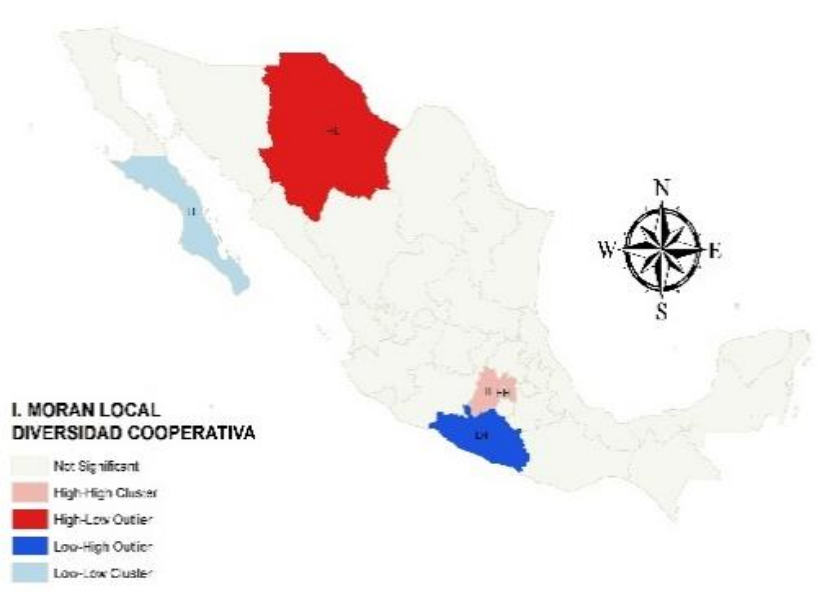

LISA para la variable de Krugman (Índice Especialización Cooperativa)

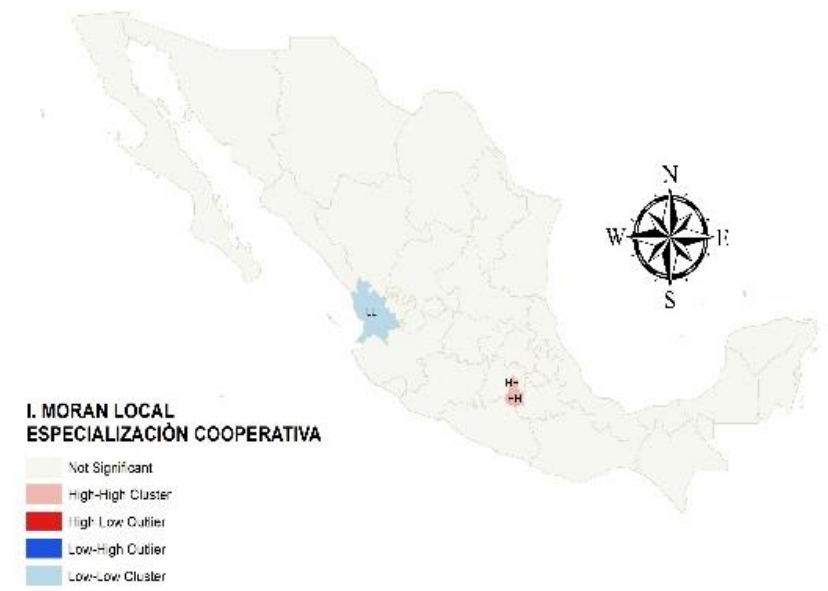

Fuente: Elaboración propia.

\section{Conclusiones}

Este trabajo provee evidencia empírica haciendo un análisis de la concentración, diversidad y especialización de los sectores cooperativos en México para el año 2019. Tema que previamente no había sido abordado en la literatura de economía social y solidaria en nuestro contexto.

Las estimaciones realizadas permitieron conocer los rankings de dichas métricas espaciales básicas, mientras que la matriz de especialización pudo reflejar concretamente cuales son los sectores cooperativos en los que cada entidad tiene una presencia comparativa revelada.

La red del espacio de los sectores cooperativos permite también identificar las proximidades de dichos sectores, mostrando que por ejemplo un sector muy tradicional como el "agro" coocurre con sectores menos tradicionales y más complejos, lo que implica que estos sectores cooperativos comparten ciertos factores (conocimiento, infraestructura, factores institucionales o sociales) entre sí. En este punto es importante señalar que las estadísticas referentes al empleo cooperativo por sectores serían útiles para contrastar los resultados de la presente investigación y verificar si el relacionamiento de unidades cooperativas es consistente con el de empleabilidad cooperativa, ya que éste último hablaría de la disposición de capacidades, con las cuales es más probable diversificar la economía cooperativa hacia otro sector adyacente. Ya que es ampliamente reconocido que una economía más diversificada puede favorecer el establecimiento de una sociedad pluralista con múltiples ocupaciones y una mejor distribución del poder económico y político (Hartmann, 2014). En ese sentido, es conveniente reflexionar sobre la falta de diversificación y especialización del cooperativismo en algunas de las entidades de nuestro país.

Por otra parte, con lo referente a la aplicación de un análisis exploratorio de datos espaciales (ESDA), con las técnicas denominadas: Índice de Moran e Índice Local de Asociación Espacial (LISA). La primera logró explicar que a nivel general la concentración cooperativa muestra un patrón espacial aleatorio, mientras que la diversidad y especialización reflejan una distribución agrupada (clusters), se pudo mapear por quintiles ambas variables y de esta manera identificar en lo general las regiones en donde se ve favorecida y desfavorecida la diversidad y especialización cooperativa, lo cual resulta relevante para la formulación de políticas regionales. Posteriormente gracias a LISA se pudo explicar localmente el nivel de significancia estadística de los agrupamientos espaciales del mismo fenómeno, teniendo como resultado que para efectos de la diversidad y especialización cooperativa las zonas calientes (favorables) son: Ciudad de México, Estado de México y Morelos; ubicadas en la zona centro del país. Mientras que las zonas frías (desfavorables) son: Baja California Sur y Nayarit. Los puntos atípicos en cuanto a diversidad cooperativa se ubican en Chihuahua y Guerrero, la primera entidad asume valores altos rodeada de entidades con valores bajos de diversificación cooperativa, mientras que la segunda entidad manifiesta el comportamiento contrario.

Se concluye concretamente que la diversidad y especialización cooperativa tienen una autocorrelación espacial positiva y además la especialización cooperativa muestra un correlación positiva y significativa con variables relacionadas con los resultados esperados de la economía social. En otras palabras, el fomentar la especialización cooperativa puede ser clave para impulsar la economía social y solidaria en el país y sus 
beneficios esperados. Más no es mejor en materia de "cantidad de cooperativas", pues vemos que la región sureña del país es la que cuenta con mayor cuantía de cooperativas, pero también es la región con menos desarrollo social y económico. Lo anterior concuerda con lo postulado por Tacchela et al. (2012), quienes sostiene que hay evidencia del papel de liderazgo de la diversificación con respecto a la ventaja competitiva (especialización) y en ese sentido, es importante resaltar que el objetivo de la especialización inteligente (incluida la especialización cooperativa) no es hacer que la estructura económica de las regiones sea más especializada (es decir, menos diversificada), sino aprovechar las fortalezas existentes, identificar oportunidades ocultas y generar plataformas novedosas sobre las cuales las regiones pueden construir una ventaja competitiva de alto valor (Balland et al., 2019).

Adicionalmente, es importante reflexionar que hoy en día emergen "nuevos sectores cooperativos" como por ejemplo el cooperativismo de plataforma, el cual es generado por los actores del sector social de la economía, y es pensado desde su concepción de una manera digital, mayoritariamente a través de apps, pero también de todas aquellas que se valen del internet y del entorno digital para operar. En el cooperativismo de plataforma, el modelo es idéntico a las plataformas conocidas para pedir transporte o alimentos desde tu teléfono, pero que en su base se considera el trabajo y bienestar de todas las personas que intervienen en el proceso ya que se basa en los principios cooperativos entre ellos el de copropiedad y gobernanza democrática (INAES, 2020). Las cooperativas de plataformas abren los mercados a otras formas de economía, evitan la monopolización de los sectores y generan estructuras sociales digitales que son más flexibles, adaptables y justas (PescaData, 2021).

Finalmente, resulta imperante que los datos derivados de los sectores cooperativos sigan siendo compilados. Sin embargo, se enmarca la necesidad que dichos datos tengan una mayor granularidad y contemplen número de socios, género, y datos adicionales que permitan realizar estudios a mayor profundidad y especificidad. Sobre todo, que los datos actuales puedan ser democratizados y abiertos al público en general, será un buen inicio para poner en el radar la importancia de las estadísticas relacionadas con la economía social y especialmente con las cooperativas en México y de esta forma seguir la "recomendación sobre la promoción de las cooperativas no. 193 adoptada por la OIT en 2002, la cual establece que las políticas nacionales deben buscar mejorar las estadísticas nacionales sobre cooperativas con miras a la formulación e implementación de políticas de desarrollo" (COPAC, 2019). Se considera necesario también que México adopte las directrices sobre estadísticas de cooperativas que en el año 2018 fueron enmarcadas por la Organización Internacional del Trabajo.

\section{Referencias bibliográficas}

ACI (2018) Datos y Cifras. Acceso en línea https://www.ica.coop/es/cooperativas/datos-y-cifras.

ArcGis (2021) Cómo funciona el análisis espacial. Acceso en línea: https://pro.arcgis.com/es/pro-app/latest/toolreference/spatial-statistics/h-how-spatial-autocorrelation-moran-s-i-spatial-st.htm.

Anselin, L. (2013) Spatial Econometrics: Methods and Models (Vol. 4). Springer Netherlands. https://doi.org/10.1007/978-94-015-7799-1.

Austin, J. E., et al. (2006) Gestión efectiva de emprendimientos sociales: Lecciones extraídas de empresas y organizaciones de la sociedad civil en Iberoamérica. Estados Unidos de América: Banco Interamericano de Desarrollo.

Alarcón, R. A. R. (2018) Dominio, declinación y fracaso de las cooperativas camaroneras en Mazatlán (México) 19822017. Pilquen, Ciencias Sociales, vol. 21, no. 15.

Araujo Batlle, A., Serrano Robles, E., \& Jordan Vallverdú, V. (2020) La presencia de las cooperativas de Catalunya en Internet. CIRIEC-España, Revista de Economía Pública, Social y Cooperativa, $\quad \mathrm{N}^{\circ} \quad 99,37$. https://doi.org/10.7203/CIRIEC-E.99.16902.

Balland, P.A. (2017) EconGeo: Computing Key Indicators of the Spatial Distribution of Economic Activities, R package version 1.3: https://github.com/PABalland/EconGeo.

Balland, P.-A., Boschma, R., Crespo, J., \& Rigby, D. L. (2019) Smart specialization policy in the European Union: Relatedness, knowledge complexity and regional diversification. Regional Studies, 53(9), pp. 1252-1268. https://doi.org/10.1080/00343404.2018.1437900.

Cabrera-Ramírez, A. K. (2015) Trascendencia de las cooperativas en México: Análisis documental del impacto socioeconómico en comunidades rurales. 17.

Cano-Salgado, M. P. C., Baltazar, E. B., \& Barba, E. (2012) Innovación social y capacidad de organización de las cooperativas pesqueras en el municipio de Balancán, Tabasco, México. Estudios Sociales, 32.

Celemín, J. P. (2009) Autocorrelación espacial e indicadores locales de asociación espacial. Importancia, estructura y aplicación. Revista Universitaria de Geografía, 18(1), pp. 11-31. [fecha de Consulta 3 de Octubre de 2021]. ISSN: 0326-8373. Disponible en: https://www.redalyc.org/articulo.oa?id=383239099001.

Ceballos, R., Olivares, M. \& Castillo A. (2016) Creación del padrón de entidades cooperativas del estado de Quintana Roo, México. Revista Global de Negocios, 4, ISSN: 2328-4668. 
COPAC (2019) The Guidelines concerning Statistics of Cooperatives. COPAC COOP. Disponible: http://www.copac.coop/the-guidelines-concerning-statistics-of-cooperatives/.

Cruz-Coria, E., Velázquez-Castro, J., \& Briones-Juárez, A. (2020) Prácticas de economía solidaria en las cooperativas artesanales de Campeche, México (Solidarity economy practices in the artisan cooperatives to Campeche, Mexico). 26.

Cutrini, E. (2009) Using entropy measures to disentangle regional from national localization patterns. Regional Science and Urban Economics, 39(2), pp. 243-250. https://doi.org/10.1016/j.regsciurbeco.2008.08.005.

Díaz de León, D., \& Rivera, I. (2019) Desafíos en el Emprendimiento en Cooperativas de la Ciudad de México: Projectics / Proyéctica / Projectique, ${ }^{\circ} 23(2)$, pp. 63-77. https://doi.org/10.3917/proj.023.0063.

Fracasso, A., \& Vittucci Marzetti, G. (2018) Estimating dynamic localization economies: The inadvertent success of the specialization index and the location quotient. Regional Studies, 52(1), pp. 119-132. https://doi.org/10.1080/00343404.2017.1281388.

Hartmann. (2014) Economic Complexity and Human Development. How Economic Diversification and Social Networks Affect Human Agency and Welfare. https://www.researchgate.net/publication/292980558_Economic_Complexity_and_Human_Development_How_Eco nomic Diversification and Social_Networks_Affect_Human_Agency and Welfare.

Hernández Herrera, C. A., Sánchez Rodríguez, S., \& Díaz Fragoso, O. (2018) Empoderamiento y cooperativismo femenino, tres estudios de caso de cooperativas lideradas por mujeres en la Ciudad de México. Acta Universitaria, 28(5), pp. 72-83. https://doi.org/10.15174/au.2018.1642.

Hidalgo, C. A., Balland, P.-A., Boschma, R., Delgado, M., Feldman, M., Frenken, K., Glaeser, E., He, C., Kogler, D. F., Morrison, A., Neffke, F., Rigby, D., Stern, S., Zheng, S., \& Zhu, S. (2018) The Principle of Relatedness. En A. J. Morales, C. Gershenson, D. Braha, A. A. Minai, \& Y. Bar-Yam (Eds.), Unifying Themes in Complex Systems IX (pp. 451-457). Springer International Publishing. https://doi.org/10.1007/978-3-319-96661-8 46.

Hidalgo, C. A. (2021) Economic complexity theory and applications. Nature Reviews Physics, 3(2), pp. $92-113$. https://doi.org/10.1038/s42254-020-00275-1.

Hoover, E.M. (1936) The Measurement of Industrial Localization, The Review of Economics and Statistics 18 (1), pp. $162-171$.

INAES (2020) Cooperativismo de plataforma. Disponible: https://www.gob.mx/inaes/es/articulos/cooperativismo-deplataforma?idiom=es.

INAES (2020) Guía: Cooperativas de energía sustentable en México. Disponible: https://www.gob.mx/inaes/documentos/guia-cooperativas-de-energia-sustentable-en-mexico.

INEGI. (2014) Censos Económicos 2014. CE. Censos Económicos 2014. CE; Instituto Nacional de Estadística y Geografía. INEGI. https://www.inegi.org.mx/programas/ce/2014/.

INEGI. (2019) Censos Económicos 2019. CE. Censos Económicos 2014. CE; Instituto Nacional de Estadística y Geografía. INEGI.

INEGI (2011) Laboratorio de microdatos y acceso remoto. Disponible: https://www.inegi.org.mx/app/microdatos/solicitud/login.aspx.

Izquierdo, M. (2021) Cooperativas e inclusión en la Ciudad de México. Deusto Estudios Cooperativos, 12, pp. 79-99. https://doi.org/10.18543/dec-12-2019pp79-99.

Krugman P. (1991) Geography and Trade, MIT Press, Cambridge

Lara, G. \& Pérez, C. (2016) El cooperativismo y el nivel socio-tecnológico en España. Revista Electrónica Nova Scientia, 8, pp. 31-42.

Lara Gómez, G. (2018) La equidad horizontal en la tributación de las cooperativas en México. Boletín de la Asociación Internacional de Derecho Cooperativo, 53, pp. 251-253. https://doi.org/10.18543/baidc-53-2018pp251-253.

Lara Gómez, G. (2020) Las Cooperativas como Entidades con Fines No Lucrativos en México: regulación y características tributarias. REVESCO. Revista de Estudios Cooperativos, vol. 134, e69161. https://dx.doi.org/10.5209/REVE.69161.

Lara Gómez, G., \& Rico Hernández, A. (2011) La contribución de las cooperativas de ahorro y crédito al desarrollo local en Querétao, México. REVESCO. Revista de Estudios Cooperativos, No 106, pp. 121-149. https://doi.org/10.5209/rev REVE.2011.v106.37376.

Luvián Reyes, G., Rosas Baños, M., \& Ramírez Vanoye, E. (2019) Pequeñas cooperativas de producción exitosas de la Ciudad de México: perspectivas y estrategias de los socios en la modernidad capitalista. Otra Economía, 12(22), pp. 42-65. Recuperado a partir de https://www.revistaotraeconomia.org/index.php/otraeconomia/article/view/14795.

Méndez-Reyes, J. (2017) Desarrollo, movilidad y economía social en Baja California: El caso de las cooperativas de transporte entre las décadas de 1930 a 1950. América Latina en la Historia Económica. https://doi.org/10.18232/alhe.853.

OIT (2015) Las cooperativas y los objetivos de desarrollo sostenible, Debate sobre el desarrollo después de 2015. Organización Internacional del Trabajo. Acceso en línea https://www.ilo.org/wcmsp5/groups/public/---ed_emp/--emp_ent/---coop/documents/publication/wcms 307228.pdf.

OIT (2017) Use of statistics on cooperatives in national policy making. International Labour Office - Geneva: ILO.

ONU (2012) 2012 Año Internacional de las cooperativas. Acceso en línea https://www.un.org/es/events/coopsyear/\#: :text=La\%20Asamblea\%20General\%20de\%20las,empleos \%20y\%20la $\% 20$ integraci\%C3\%B3n\%20social. 
Pérez González, M. del C., \& Valiente Palma, L. (2019) Territory and cooperativism: A spatial analysis of the Spanish region of Andalusia. Economics \& Sociology, 12(2), pp. 265-283. https://doi.org/10.14254/2071-789X.2019/12-2/16. PescaData. (2021) El cooperativismo de plataforma. Disponible: https://pescadata.org/cooperativismo-de-plataforma/.

Rojas-Herrera, J. J. (2013) Panorama general del cooperativismo agropecuario en México. Estudios Agrarios, vol. 19, pp. 121-138.

Rogerson, Peter, y Peter Kedron (2012) Optimal Weights for Focused Tests of Clustering Using the Local Moran Statistic, Geographical Analysis, 44(2), pp. 121-133. https://doi.org/10.1111/j.1538-4632.2012.00840.x.

Rosales, G. C., \& Fuentes, E. (2017) Índices de concentración y especialización de la producción agropecuaria en los estados mexicanos para los años 1993, 1998, 2003, 2008 y 2013. Revista mexicana de agronegocios, vol. 41 pp. 696708.

Tacchella, A., Cristelli, M., Caldarelli, G. et al. (2012) A New Metrics for Countries' Fitness and Products' Complexity. Sci Rep 2, 723 https://doi.org/10.1038/srep00723.

Tirole, J. (2017) La Economía del Bien Común. México: Taurus.

Saviotti, P. P., \& Frenken, K. (2008) Export variety and the economic performance of countries. Journal of Evolutionary Economics, 18(2), pp. 201-218. https://doi.org/10.1007/s00191-007-0081-5.

Sosa Gonzalez, J. L. S. (2020) Diagnóstico multidimensional y morbilidad de las cooperativas de producción en México: Un estudio descriptivo y explicativo de las áreas de oportunidad de las cooperativas de la región de la costa de Oaxaca. Boletín de la Asociación Internacional de Derecho Cooperativo, 57, pp. 309-350. https://doi.org/10.18543/baidc-572020pp309-350.

Sosa González, J. L. S., Gómez Abad, P., Carmona Silva, J. L., \& Medel Sánchez, J. M. (2019) Una aproximación empírica a la viabilidad de los emprendimientos sociales en México: El ciclo de vida de las cooperativas de la Región de la Costa de Oaxaca. REVESCO. Revista de Estudios Cooperativos, Segundo Cuatrimestre, No 131, pp. 151-178. https://doi.org/10.5209/REVE.63564.

Soto Alarcón, J. M., González Gómez, D. X. (2020) Feminist political ecology and rural women-led cooperatives in Hidalgo, Mexico. Textual, 75, pp. 131-155. https://doi.org/10.5154/r.textual.2020.75.06. 